\title{
An Efficient Deployment Model for Maximizing Coverage of Heterogeneous Wireless Sensor Network Based on Harmony Search Algorithm
}

\author{
Belal Al-Fuhaidi $\mathbb{D}$, Abdulqader M. Mohsen $\mathbb{D}$, Abdulkhabeer Ghazi $\mathbb{D}$, \\ and Walid M. Yousef $(\mathbb{C}$
}

Faculty of Computing and IT, University of Science and Technology Sana'a, Yemen

Correspondence should be addressed to Belal Al-Fuhaidi; belalarh@gmail.com

Received 15 July 2020; Revised 13 October 2020; Accepted 22 October 2020; Published 23 November 2020

Academic Editor: Eduard Llobet

Copyright ( $\odot 2020$ Belal Al-Fuhaidi et al. This is an open access article distributed under the Creative Commons Attribution License, which permits unrestricted use, distribution, and reproduction in any medium, provided the original work is properly cited.

\begin{abstract}
Due to the increase of Wireless Sensor Network (WSN) technologies demand, the optimal sensor node deployment is considered as one of the most important factors that directly affect the network coverage. Most researches in WSNs that solved the problem of coverage in homogeneous and heterogeneous cases are suffering from many drawbacks such as consumed energy and high cost. In this paper, we propose an efficient deployment model based on probabilistic sensing model (PSM) and harmony search algorithm (HSA) to achieve the balance between the network coverage performance and the network cost in a heterogeneous wireless sensor network (HEWSN). The HSA is used for deployment optimization of HEWSN nodes which makes a balance between the coverage and financial cost. The PSM is used to solve the overlapping problem among the sensors. The performance of the proposed model is analyzed in terms of coverage ratio and cost evaluations. The simulation results showed the capability of the proposed heterogeneous deployment model to achieve maximum coverage and a minimum number of sensors compared to homogeneous deployment. Furthermore, a comparative study with a meta-heuristic genetic-based algorithm in HEWSN has also been conducted, and its results confirm the superiority of the proposed model.
\end{abstract}

\section{Introduction}

Over the past decade, wireless sensor networks (WSNs) have become a wealthy research field, providing several new trends of applications. Nowadays, WSNs are considered an integral part of the Internet of Things (IoT) systems [1]. WSNs are set of low-power, low-cost, small size, and multifunctional sensor nodes that work together to sense the field, perform data processing, and communicate over AoI wirelessly. Nodes send the data aggregation to a specific station called sink or data sink, which is used to send information outside WSN to the end-user as shown in Figure 1 [2].

Current applications of WSNs include the applications that use WSN in physical life, such as military, civilian, connected vehicles, smart cities, smart grids, smart healthcare, networks of robots, and disaster recovery network civilian. For example, in the military, the WSN is used for targeting system and battlefield surveillance. In the civilian application, WSN can monitor building, traffic, environment, wildlife, security, smart agriculture system, and several other applications [3,4]. The design of several WSN applications requires deciding an exact place of the optimal positions of sensor nodes to be located in AoI [5].

Maximizing the coverage area of the WSN system has been a current research area of WSN in the last years. Actually, WSN depends upon coverage of the network that is basically depending upon the deployment of sensor nodes [6]. The coverage area has several types based on the observed field such as the barrier coverage (means how can to protect the area from an intruder), point coverage (means how to cover a group of points), and full coverage (means that every 
point in the AoI is covered by at least one sensor). In each coverage type, the quality of each point can be assessed after the deployment process $[7,8]$.

The deployment problem of WSN is a critical issue because it affects both the overall performance and the energy consumed by the sensors in the whole system $[9,10]$. The deployment scheme is constructed according to many factors such as application, kind of sensor, and environment of the sensor [11]. Sensors can be deployed in AoI either deterministically, i.e., planned or randomly [12]. The planned sensor deployment is applied in many studies. For example, Authors in [10] proposed a novel algorithm in planned deployment was called maximal coverage hybrid search algorithm to achieve maximum coverage with a minimum number of deployment sensors. However, this type can be used for the placement of sensor nodes in the small-scale area and is not practical to cover AoI such as hostile environments and large regions. The second type of WSN deployment is called random sensor deployment, which is the most popular deployment strategy and can be considered in this paper. Dynamic deployment was used to enhance coverage in WSNs by redistribution sensor nodes after initial random deployment [13]. In the same view, for the sensor localization, a novel localization methods using dynamic deployment, collection of a novel local search process, and HS algorithm was used in [14].

Further, the deployment states are divided into four states: predeployment, initial deployment, postdeployment, and redeployment. The predeployment and initial deployment are using the manual placement of the nodes by a human or a robot or launching them from a plane. The redeployment was used in adding new nodes to the network to replace some broken. A postdeployment phase is used when the network topology has been evolved due to a relocation of nodes $[15,16]$. According to the density, a sensor network deployment could be a sparse or dense deployment in which low or high number of sensors is used to cover AoI, respectively. The sparse deployment type is used when obtaining maximum coverage using less number of nodes. The dense sensor deployment type usually has more number of nodes in the AoI than required [17].

In general, WSN environments can be categorized as heterogeneous or homogeneous wireless sensor nodes. The homogenous wireless sensor nodes include sensors that have the same sensing range, communication range, and the same energy. The networks having the sensors which have various sensing range, communication range, network protocols, or different power are named heterogeneous sensor nodes. Heterogeneous sensor deployment has many features, such as it can obtain a more effective and flexible network. Also, it can increase network performance and takes into account reducing the cost of sensor deployment, energy, and communication [7, 18-20].

Maximum coverage sensor deployment problem presented with different exact and meta-heuristic algorithms. The meta-heuristics are most widely used. The harmony search algorithm is a recent meta-heuristic algorithm which was invented by [21]. HSA has successfully applied to solve a wide range of NP-complete optimization problems [22]. It is

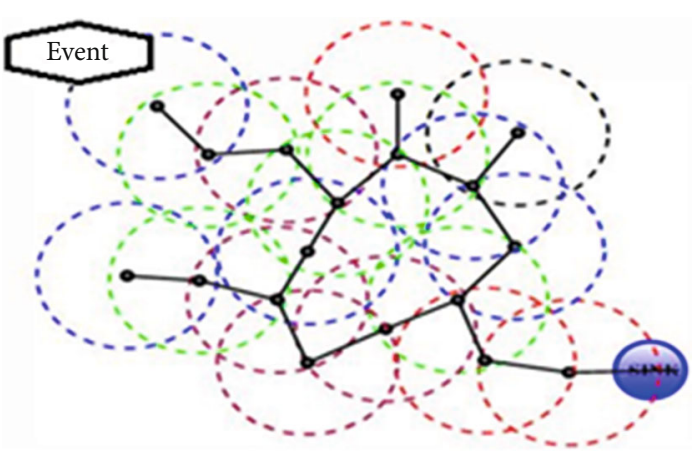

Figure 1: A general architecture for WSN.

inspired by the natural musical performance procedures that occur when a musician searches for a better state of harmony. According to [23], HSA has many features in comparison with traditional optimization techniques can be summarized as follows:

(i) Fewer mathematical requirements (low amount of computation), free divergence

(ii) HSA may overcome the limitation of GA by considering the relationship among all decision variables, whereas the GA only considers the relationship of only two parents in each enhancement

Therefore, HSA in HEWSN will be a good alternative to solve the coverage problem. Moreover, it can make a balance between maximizing the coverage and reduction of both financial cost and unnecessary energy consumption.

The optimization of random deployment problem is the critical issue in WSN applications that required the optimization of maximum coverage with minimum deployment cost. The optimization problem for coverage is related to power saving, network reconfiguration, and cost-minimizing. The deployment cost of the sensing nodes is the major factor in WSNs. WSN is a combination of many sensors where's each sensor node has a limited radion range. Thus, the researcers in [24] studied the optimum numbers of sensors that can reduce the network cost and maximize the coverage efficiently.? Although maximum coverage sensor deployment with minimum cost in WSNs has often been investigated in many studies [20], the performance of coverage is still unbalanced and suffering from many drawbacks such as energy and financial cost. In addition, research on this problem in HEWSN has progressed at a slow pace. In several WSN applications, the constructed nodes are provided with many types of sensors to provide various sensing services which can be achieved by heterogeneous sensors only [25]. Moreover, using an optimal mixture of some inexpensive low capability devices and some expensive high-capability devices can significantly enhance the performance and coverage and extend the duration of a network's lifetime [20,26,27].

In this paper, we consider the problem of maximizing coverage area in heterogeneous WSN with an optimized sensor deployment and minimization in sensors overlapping. The main contribution of this paper is summarized as: 
(1) We propose an efficient deployment model in HEWSN which gives a maximum coverage area with the optimized number of sensor nodes (network cost)

(2) In this model, we develop the PSM of the homogenous WSN in [11] to minimize the overlapping problem in HEWSN and explore the ability of the modified HS algorithm in providing solutions for HEWSN coverage problems in a way that the optimal deployment sensor nodes such that the network cost can be minimized and the coverage requirements can be satisfied

(3) A modified objective function based on HEWSN combines coverage ratio, the number of sensor nodes with different sensing range, and the minimum distance between sensor nodes is proposed to support the selection of the optimal number of sensor nodes and their locations

(4) This model which consists of PSM and HS algorithm is the first that was used in HEWSN deployment, and the comparative study is conducted with a homogeneous deployment using HS algorithm and a metaheuristic genetic-based algorithm in HEWSN using different performance analysis including maximum coverage, network cost, and overlapping effect

The rest of the paper is organized as follows. Section 2 introduces the literature reviews. The proposed HEWSN deployment model and problem definition are described in detail in Section 3. In Section 4, the experimental results and comparative study with those of other state-of-the-art methods are explained in detail. The conclusions of this study are presented in Section 5.

\section{Literature Review}

The research works classified the deployment environment to HEWSN and HOWSN deployments. Actually, most studies in WSNs have focused on the deployment of many numbers of inexpensive homogenous sensor devices. However, in practical settings, it is often suitable to consider HEWSN deployment that has various capabilities [26]. HEWSN deployment takes into account the reducing costs of sensor deployment, energy, and communication. In addition, HEWSN deployment can be covered in a broad area and can be more efficient than HOWSN deployment. Authors in [28] tested the complete coverage problem of circular sensing footprints through various radii by two configurations of heterogeneous. In the first case, the best results are obtained when the sensing radius for one kind of nodes is approximately half of the others. The second case, including sensing cost and optimum coverage density, is obtained when the sensing radius of one disk is almost about onefifth of the other. The results showed that more efficient coverage can be obtained, and the sensing cost is significantly minimized of HEWSN deployment as compared to the HOWSN deployment case. HEWSN deployment acts also a role in improving the network's lifetime and coverage of
WSN by extending the network life cycle. The optimal HEWSN deployment can extend lifetime sensing coverage by many times as much as with HOWSN deployment. It takes into account both the initial coverage and the duration of sensing operation [29]. Authors in [30] introduced mathematical models to study the effect of HEWSN deployment on the network's coverage and energy consumption using sensors of different types. The simulation results showed maximizing coverage and energy consumption. In addition, HEWSN deployment was also used for increasing network security [31]. The authors introduced reinforced barrier-coverage with consideration of HEWSN deployment in various capabilities. It used this tool to guarantee that any penetration variation of the intruder is monitored by at least one sensor and to extend the lifetime of heterogeneous reinforced barriers.

In contrast, there is another kind of sensor called smart nodes. Smart nodes are deployed according to the distance information to maximize the coverage and the network connectivity. Authors in [32] introduced smart nodes to provide the link establishment between unconnected nodes and the sink. Simulation results proved that the coverage would be increased with a limited minimum optimum number of heterogeneous smart nodes and connectivity. Still, the smart nodes have some limitations such as an expensive cost.

In HEWSN, authors in [33] used heterogonous sensor deployment to solve the obstacles of the WSN coverage problem. They proposed a novel algorithm based on the circle packing technique. They have confirmed that the proposed algorithm avoided the obstacle, produced sensor deployments of broad coverage, and minimized the sensing range demanded for each inside sensing node for accomplishing the packing condition. Authors in [34] used GA to maximize the coverage in the heterogeneous case by removing intersection among the nodes. Experimental results showed that no intersection between the nodes and the coverage area was maximized. Authors in [35] presented PSM and an improved GA (IGA) to maximize the coverage with a number of sensors which have different sensing ranges. In addition, the definition of the overlapping for the fitness function includes in these improvements. The algorithm tested on 15 states was constructed for this problem. The simulation results showed that the proposed algorithm is effective in all terms of the computational complexity, stability, and quality of solutions. However, this improved version of GA is still suffering from high complexity, because of using many sensors with various sensing radius.

The Particle Swarm Optimization (PSO) algorithm has been applied to solve optimization problems in WSNs [20]. Authors in [36] proposed an energy-efficient and lightweight self-organized distributed greedy heuristic algorithm to maximize coverage area with a minimal number of mobile nodes. They used random deployment of nodes with sufficient node density over two-dimension areas. Simulation results showed the improved performance of the proposed technique in terms of average displacement of nodes, the number of computation rounds, and the number of active nodes. In HEWSN, Song et al. [37] proposed a distributed coverage control law with mobile sensors and PSM based on PSO. The methodology was used to minimize 
the coverage cost, as well as communication ranges, and exceed a threshold. However, they discarded the connectivity process. In fact, PSO has a high-quality solution, simplicity, and fast convergence. On the other hand, PSO has some limitations, such as using big amounts of memory which may limit its implementation to base stations [38].

Ant Colony Optimization (ACO) provided solutions to problems in HEWSN, such as [39]. The authors proposed a swarm intelligence based on the ACO method. The methodology depended on searching the maximum number of connected covers that achieve both sensing coverage and network connectivity. Simulation results showed that the proposed method provided a more approximate, effective, and efficient way for extending the lifetime of HEWSN. In the same context, Lin et al. [40] proposed an ACO technique that can maximize the lifetime of HEWSN. This technique depended on searching the maximum number of disjointassociated covers that satisfy both network connectivity and sensing coverage. Simulation results approved the effectiveness and efficiency of the technique and showed that an ACO is a promising method for extending the HEWSN lifetime.

Harmony search algorithm (HSA) is a music-based meta-heuristic algorithm for solving optimization problems. This algorithm has been proposed to solve several kinds of problems in the previous decade, generating very effective and efficient results in WSN. For the coverage problem in HEWSN, the HS algorithm is not used yet for deployment optimization in HEWSN, but is used in HOWSN in several researches. It is more efficient compared to other metaheuristic algorithms and traditional techniques that computationally expensive [23]. HSA is selected to maximize the coverage and minimize the number of deployed sensors in WSN. HSA had many providing solutions for WSN problems. For solving coverage and connectivity, Halim et al. [41] implemented modified HSA for node deployment purpose and enhancing the coverage. The modified HSA outperformed better compared to HSA such as the highest coverage achieved by modified HSA is $70 \%$ while HSA highest coverage is $54 \%$. However, it suffers from high energy cost, and the full coverage is still unreachable. Mohamed et al. [42] examined a scalable HSA in terms of $k$-coverage enhancement algorithm and execution time. The proposed algorithm tried to improve initial coverage and to achieve the required $K$ -coverage degree efficiently. Also, the same author in [13] proposed HSA based on dynamic deployment to maximize the network coverage and connectivity. Authors in [43] have proposed the combination of the Markov chain model and HSA to solve the coverage hole problem by redistribution mobile nodes after the initial deployment of the static nodes. The HSA was applied to set the optimal positions of the added mobile nodes, and the Markov chain model was applied to illustrate the network schemes. Authors in [44] also proposed HSA to enhance the connectivity of the nonuniform density WSNs. The simulation results showed an increased coverage area of the cluster nodes and network connectivity significantly.

Maximizing network lifetime and minimizing energy consumption is another problem solved by HSA in WSN.
Authors in [45] proposed a proper position for the date sink node that gathers data from whole sensors. Also, they proposed two phases in WSN using the improved HSA; the first phase is used to achieve a sensor node deployment by making the sensors power balanced in both $k$-coverage and connected WSN. The second phase tries to increase the network lifetime by moving a few of high power consumption mobile nodes to the nearest locations of low power consumption ones. Authors in [46] introduced a protocol built on HSA to cover all of the target areas by adjusting the sensing range of every sensor node. The results showed decreasing both the nodes sensing radius and energy consumption. Authors in [47] introduced both HSA and topology control protocol. This protocol has many tasks such as learning automata to set the suitable transition radius of the sensor nodes in a distributed topology environment. In addition, it is able to supply the full connectivity in sparse deployment and to extend the lifetime of the sensor network by keeping the energy consumption least. The simulation results showed the efficiency of the proposed protocol. Authors in [48] proposed a new algorithm that is named efficient energy dynamic clustering based on HSA that orders the network sensors into a suitable number of clusters. Simulation results showed that the proposed algorithm could achieve an optimal number of clusters in each round during simulation. Authors in [49] proposed combining HSA with ACO to improve the performance of each other. The role of modified HSA is to increase the network lifetime. Authors in [50] proposed an enhanced HSA in a $k$-covered and connected WSN. The objective is to achieve optimal coverage with sensor node deployment and efficient energy by staying node $k$-coverage and connectivity in hotspot areas. On the same idea, Authors in [51] examined HSA to display a solution of the coverage area targets with at least both energy consumption and $k$-sensors in the systems and compare its results with the PSO algorithm. Simulation results appeared that the HSA had more control either in minimizing the whole of sensor turns or in accomplishing $k$ -coverage and higher convergence rate than PSO. Authors in [52] proposed a shared-infrastructure deployment problem technique to maximize the coverage network, and HSA is adjusted and assigned maximum budget to introduce a solution for metropolitan WSNs. Authors in [53] proposed an HSA for choosing the optimal path in WSN to achieve objective optimization such as reducing the transmitted power consumption and the network transmission delay. An improved particle swarm optimization (PSO) combined with a mutation operator is introduced to search the parking positions with optimal coverage rate. Then, the genetic algorithm (GA) is adopted to schedule the moving trajectory for multiple mobile sinks [54].

For minimizing cost, Wang et al. [55] introduced a model called binary differential evolution harmony search to achieve many factors such as minimum cost, reliability, and scalability of industrial WSNs. The simulation results demonstrated that the improved model is efficiently valid and reliable. Authors in [11] proposed an HSA and compared their results to GA. The simulation results showed the superiority of HSA on GA for maximizing the network coverage and minimizing the number of sensor deployment by a factor 
of $40 \%$. However, it has a higher cost compared to HEWSN. Sun et al. [56] minimized cost by determining the positions of the base station, sensor nodes, and relay nodes in both single-tiered and two-tiered to meet the coverage and connectivity requirements.

Look closer to what has been so far presented in the previous studies, we find most studies in WSNs that solved the problem of coverage in homogeneous case are still suffering from many drawbacks such as high consumed energy and high financial network cost. Furthermore, and another look at the other meta-heuristic-based solutions for HEWSN presented in this section, none of them addresses the problem of finding the optimal number of sensor nodes that can minimize the network cost and at the same time maximize the network coverage. The HS-based solutions were providing optimum solutions to different problems in HOWSN, but were not included in HEWSN coverage problems. Encouraged by these finding, the proposed work requires a more balancer method between maximizing coverage and reducing unnecessary network cost with an overlapping minimization, therefore we propose a solution in HEWSN for finding the optimal number of sensor nodes (i.e., the network cost) in a way that the optimal location of these sensors can be reached (i.e., full coverage) using PSM and HS algorithm. (a) According to the three parameters of HSA, Harmony Memory Considering Rate (HMCR), Pitch Adjusting Rate (PAR), and Bandwidth ( $\left.\mathrm{BW}_{\text {range }}\right)$, the HSA can find the suitable number of sensor nodes as well as its positions. In addition, PSM can solve the overlapping problem.

A description of the proposed model and the problem formulation that is considered in this research is presented in the next section.

\section{HEWSN Deployment Model and Problem Formulation}

This section presents the block diagram of the proposed HEWSN deployment model, which is divided into four main steps. The first step overviews the general assumptions and preliminaries of the AoI initialization in subsection A. In the second step, the methodology of HEWSN random deployment is presented in subsection B. In the third step, the determination of coverage probability based on PSM is introduced in subsection C. In the last step, the HSA which is considered the optimization algorithm is presented in subsection D. Figure 2 shows the block diagram of the proposed model.

3.1. Assumptions and Preliminaries of AoI. The assumption and preliminaries of AoI are presented as follows:

(i) The AoI has 2-D, width (W), and height (H).

(ii) Two types of nodes with different sensing ranges $R_{s_{i}}$ which represent the radius of each sensor node as given in the following equation:

$$
R_{s_{i}}=f_{i}\left(\frac{\text { sensing_range }}{i}\right) i=1 \text { to } n,
$$

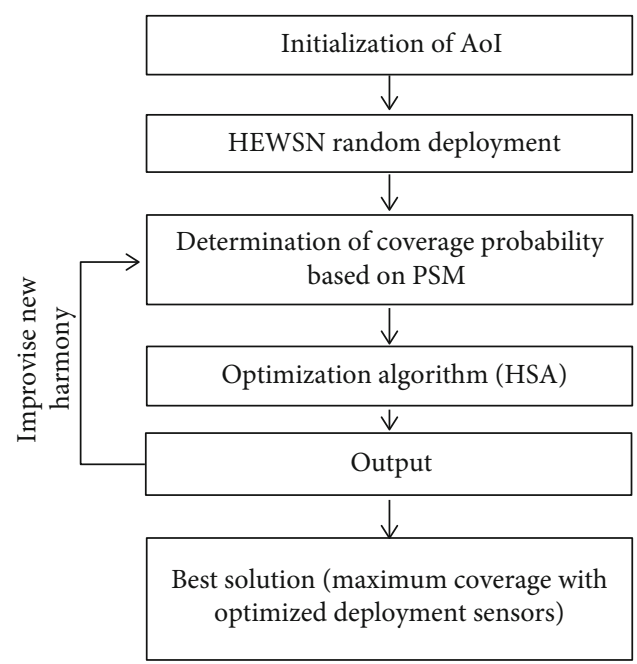

FIgURE 2: The block diagram of the proposed model.

where $f_{i}$ is the number of different sensing ranges types (i.e., if sensing range $=10$ meter, $R_{s_{1}}=10$ meter, $R_{s_{2}}=5$ meter,....etc.).

Uncertainty range represents uncertainty value in the coverage sensor detection. We assume that its value equal to half of $R_{s}$ [57] and is given in the following equation

$$
U_{e}=\frac{R_{s_{i}}}{2}
$$

(i) The AoI divided into a number of rows and columns which are represented in a group of cells

(ii) According to $[58,59]$, each cell size has the same dimensions and is set manually based on the sensor's sensing range. The number of cells in width and height of AoI is calculated according to the following equations, respectively

$$
\begin{gathered}
\text { Cells }_{W \_D i m}=\left|\frac{W}{\text { cell_width }}\right| \\
\text { Cells }_{H \_ \text {Dim }}=\left|\frac{H}{\text { cell_height }}\right|
\end{gathered}
$$

(iii) Nodes are immobile, i.e., static

The target point is the center of each cell which located at $x_{\text {th }}$ row and $y_{\text {th }}$ column as illustrated in Figure 3.

\subsection{Problem Formulation}

3.2.1. HEWSN Random Deployment. The maximum number of deployed sensors is equal to the AoI cells. Each sensor node position in AoI encodes in real number. Each position 


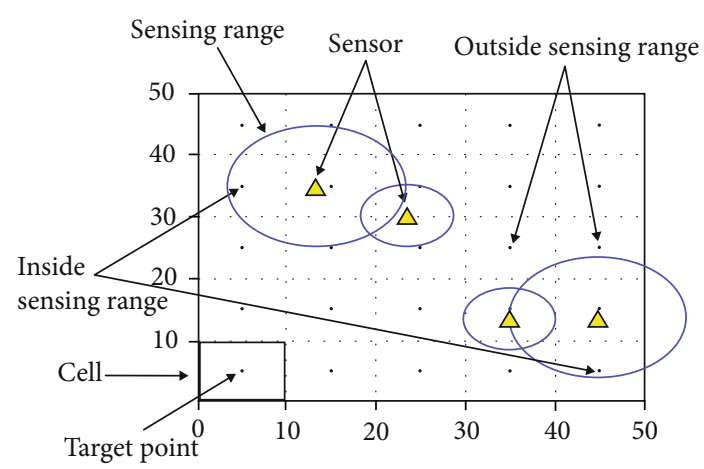

FIGURE 3: Set of target points in the area of interest $50 \times 50 \mathrm{~m}^{2}$.

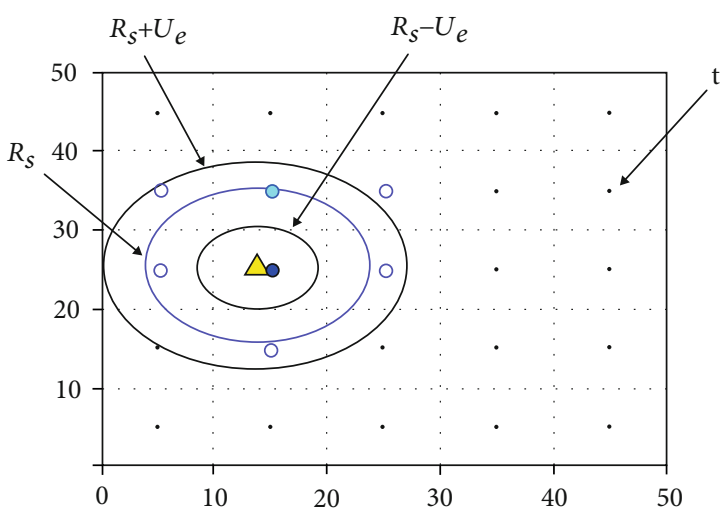

Figure 4: An explanation of probabilistic sensing model.

represents both $x$-axis and $y$-axis, which is determined under consideration of the lower and upper boundaries for each sensing node. The lower boundaries of each sensor node are shown in the following equations, respectively [11].

$$
\begin{aligned}
& \text { lower }_{x_{\mathrm{Si}}}=R_{s_{i}}-U_{\mathrm{e}}, \\
& \text { lower }_{y_{s i}}=R_{s_{i}}-U_{e},
\end{aligned}
$$

where the lower ${ }_{x \mathrm{si}}$ and lower $y_{\mathrm{si}}$ are lower boundaries of $x$ -axis and $y$-axis for every sensor node. The upper boundaries of each sensor node are defined in the following equations, respectively:

$$
\begin{gathered}
\text { upper }_{x_{\mathrm{si}}}=W-\left(R_{s_{i}}-U_{\mathrm{e}}\right), \\
\text { upper }_{y_{\mathrm{si}}}=H-\left(R_{s_{i}}-U_{\mathrm{e}}\right),
\end{gathered}
$$

where $W$ and $H$ are the dimensions of AoI, whereas upper $_{x \mathrm{si}}$ and upper $y_{\mathrm{si}}$ are upper boundaries of $x$-axis and $y$ -axis for every sensor node. The aim of boundaries calculation in both Equations (6) and (7) is to avoid sensing unnecessary field by preventing sensor nodes from being close to the sensing field border, which leads to save up energy.

In this paper, the deployment process is done randomly. So, the random positions for each sensor node are produced in the following equations.

$$
\begin{array}{r}
x s_{i}=\text { lower }_{x \mathrm{si}}+\left(\text { upper }_{x \mathrm{si}}-\text { lower }_{x \mathrm{si}}\right) \times \text { rand }() \\
y s_{i}=\text { lower }_{y_{\mathrm{si}}}+\left(\text { upper }_{y_{\mathrm{si}}}-\text { lower }_{y_{\mathrm{si}}}\right) \times \operatorname{rand}()
\end{array}
$$

where rand () is a random function used to generate numbers within $(0,1)$. However, Equations (8) and (9) are used to generate unlimited random positions. Therefore, we assume a restricted range between the minimum and the maximum number of sensors as illustrated in the following equations [11]:

$$
\begin{gathered}
\operatorname{Min} \_\mathrm{no}_{S}=\frac{W}{2\left(R_{s_{i}}+U_{e}\right)} \times \frac{H}{2\left(R_{s_{i}}+U_{e}\right)}, \\
\operatorname{Max} \_\mathrm{no}_{S}=\frac{W}{2\left(R_{s_{i}}-U_{e}\right)} \times \frac{H}{2\left(R_{s_{i}}-U_{e}\right)},
\end{gathered}
$$

where $R_{s_{i}}$ is the sensing range, $U_{e}$ is the uncertainty range, and both were calculated in Equations (1) and (2), respectively. Doubling the values in Equations (10) and (11) is necessary to gain the diameter of $R_{s_{i}}$ and $U_{e}$ of each sensor.

\subsubsection{Categorization of Coverage Probability Based on PSM.} In WSN, the best signal of the sensor node is based on the sensing range. There are two popular types of sensing models, which are used to enhance the sensing range in WSN. The first model is called Binary Sensing Model (BSM), and the second model is called Probability Sensing Model (PSM) [60]. The BSM supposed that the sensor node can detect each point if the point is within its sensing range. On the other hand, PSM, which is considered more realistic and more precise than BSM, takes in practice several dependent factors instead of the sensing radius that is used in BSM. These factors have no associated uncertainty in BSM such as noises and interferences among sensors that can affect the coverage value negatively and minimize the signal intensity $[61,62]$. The coverage probability of PSM is shown in Figure 4 and calculated in Equation (12) [60].

$$
\operatorname{Prob}_{\mathrm{cov}}\left(s_{i}, \boldsymbol{t}\right)= \begin{cases}1 & \text { if } d\left(s_{i}, \boldsymbol{t}\right) \leq R_{s_{i}}-U_{e}, i=1 \text { to } n, \\ e^{-\left(\frac{\alpha_{1} \lambda_{1}}{\lambda_{2} \beta_{2}}+\alpha_{2}\right)} & \text { if } R_{s_{i}}-U_{e}<d\left(s_{i}, \boldsymbol{t}\right)<R_{s_{i}}+U_{e}, \\ 0 & \text { if } d\left(s_{i}, \boldsymbol{t}\right) \geq R_{s_{i}}+U_{e},\end{cases}
$$

where $R_{s_{i}}$ and $U_{e}$ are calculated according to Equations (1) and (2), whereas $d\left(s_{i}, \boldsymbol{t}\right)$ is the calculated distance between the position of the sensor node $s_{i}$ and the position of target point $t$ in the AoI. This distance is considered the real Euclidean distance between $s_{i}$ and $\boldsymbol{t}$ in AoI as shown in Equation (13) [2]: $R_{s}$.

$$
\boldsymbol{d}\left(\boldsymbol{s}_{i}, \boldsymbol{t}\right)=\boldsymbol{Q}_{i}\left(\sqrt{\left(x_{s i}-x_{t}\right)^{2}+\left(y_{s i}-y_{t}\right)^{2}}\right), \quad i=1 \text { to } n,
$$

where $\boldsymbol{Q}_{\boldsymbol{i}}$ is the different sensing ranges that used for 


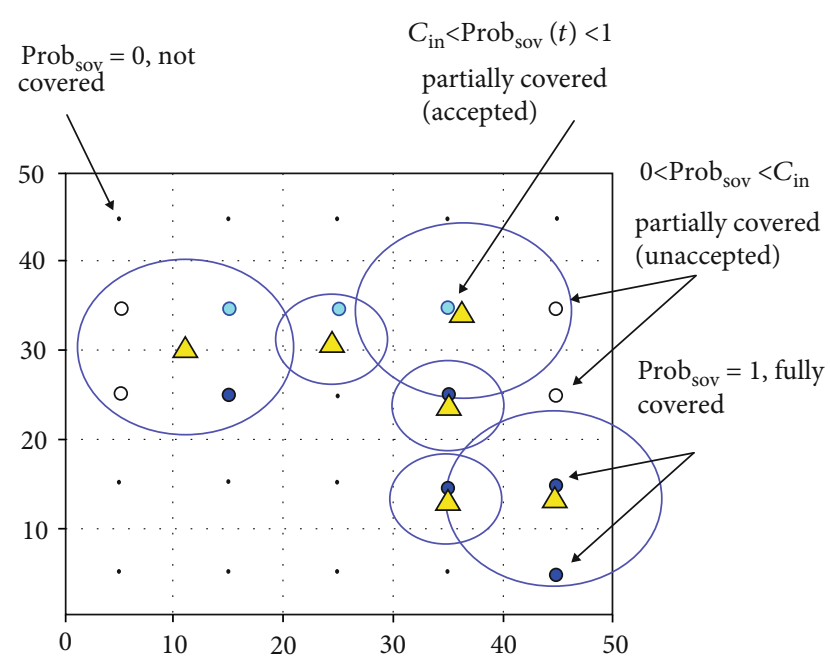

FIGURE 5: Categorization of target points based on the coverage probability of PSM.

covering the AoI, whereas $x$ and $y$ are the coordinates for each $s_{i}$ and $t$. The parameters $\alpha_{1}, \alpha_{2}, \beta_{1}$, and $\beta_{2}$ are representing the physical characteristics of the sensor unit and are supposed to be: $\alpha_{1}=1 ; \alpha_{2}=0 ; \beta_{1}=1$; and $\beta_{2}=0.5$ for each type, whereas $\lambda_{1}$ and $\lambda_{2}$ are considered as input parameters and calculated by the following equations $[57,62,63]$.

$$
\begin{gathered}
\lambda_{1}=U_{e}-R_{s_{i}}+d\left(s_{\mathrm{i}} ; t\right), \\
\lambda_{2}=U_{e}+R_{s_{i}}-d\left(s_{\mathrm{i}} ; t\right) .
\end{gathered}
$$

In PSM, the overlapped region occurs when the target point $t$ exists in the overlapping region for the set of sensors $S_{\mathrm{ov}}$. Thus, the probability of overlapping coverage in PSM would be calculated by the following equation [62].

$\operatorname{Probs}_{\mathrm{ov}}(t)=1-\prod_{s_{i} \in S_{o v}}\left(\mathrm{e}^{-} \frac{\left(\alpha_{1} \times \lambda_{1}^{\beta_{1}}\right)}{\lambda_{2}^{\beta_{2}}}+\alpha_{2}\right), \quad i=1$ to $n$,

where $s_{i}$ is the sensor type. The $\operatorname{Probs}_{\mathrm{ov}}(t)$ value was based on a coverage threshold value $\left(C_{\text {th }}\right)$ and was divided into four categories:

(1) If $\operatorname{Probs}_{\mathrm{ov}}(t)=1$, the target point is completely covered as illustrated in Figure 5 in a blue dark circle (acceptable)

(2) If $C_{\mathrm{th}}<\operatorname{Probs}_{\mathrm{ov}}(t)<1$, i.e., the target point is (partially covered-considered covered), as illustrated in Figure 5 in a sky blue circle (acceptable)

(3) If $0<\operatorname{Probs}_{\mathrm{ov}}(t)<C_{\mathrm{th}}$, i.e., the target point is (partially covered-considered uncovered), as illustrated in Figure 5 in a blank circle (rejected)

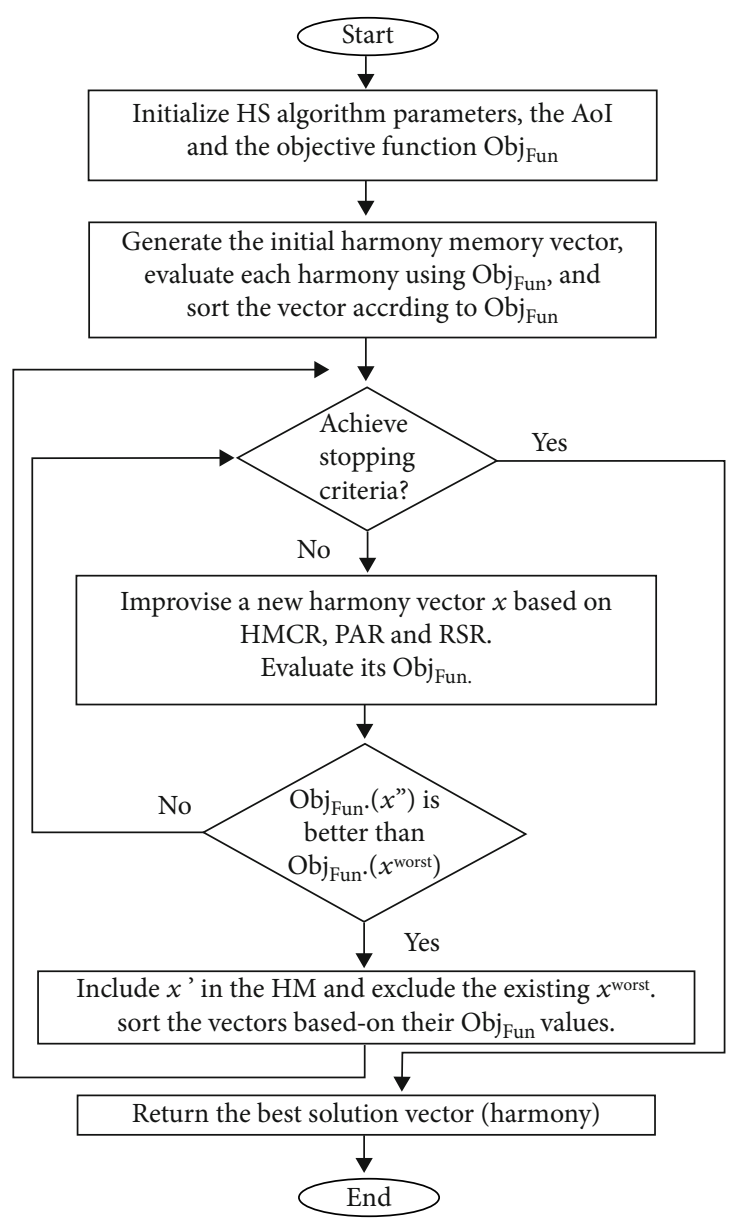

Figure 6: Steps of harmony search algorithm.

(4) If $\operatorname{Probs}_{\mathrm{ov}}(t)=0$, the target point is completely uncovered, as illustrated in Figure 5 in a filled circle (rejected)

3.3. Harmony Search Optimization. In this section, we introduce an engineering approach applied in the design and development of an algorithm, inspired by the improvisation process of the musical instruments to find fantastic harmony, called harmony search algorithm. It has received a great deal of attention regarding its potential as an optimization technique for solving discrete and continuous optimization problems. In HS, harmony parameters are typically used to create new harmony in each improvisation. The central role of these parameters is to direct the search toward the most desirable areas of the search space. These parameters are

(1) Harmony memory size (HMS) representing the total number of harmonies in the HM

(2) Harmony memory consideration rate (HMCR) which represents the probability of picking up values from $\mathrm{HM}$ to the variables in the solution vector

(3) Random selection rate (RSR) representing the probability of randomly chosen feasible values from the 
range of all possible values to the variables in the solution vector, formally

(4) Pitch adjusting rate (PAR) representing the probability of further adjusting the pitch with neighboring pitches

(5) The number of improvisations (NI) representing the number of iterations to be used during the solution process, or stopping criterion

HS algorithm starts with an initial HM of $\$ \mathrm{n} \$$ harmonies generated randomly. Each harmony in the harmony memory represents a potential solution to the problem under consideration. These solution vectors are represented by the number of sensor node positions which are represented in real numbers. For HEWSN, the solution vectors are produced from AoI dimensions randomly and kept in HM. Each harmony in the harmony memory is evaluated using an objective function. The objective function used to measure the better quality of the solution vector is described in Subsection 3.3.1. The harmonies evolve through successive iterations, called improvisations. During each improvisation, a new harmony is created through harmony operators. After that, the harmony memory is updated if the new harmony is better than its worst one. The procedure continues until the termination condition is satisfied. When the termination condition is satisfied, the best harmony obtained is regarded as an optimal or approximate optimal solution to the problem. Figure 6 shows the optimization steps of HS, which is presented in detail in the next subsections.

3.3.1. Initialize the Algorithm and Problem Parameters. HS algorithm parameters that are required to solve the optimization problem (i.e., HMS, HMCR, PAR, BW, and NI) are specified in this step. These parameters are used to improve the solution vector. The probability of performing HMCR operation is chosen in such a way so that the inheritance of existing strings in HM occurs without disruption. Generally, HMCR rate lies between 0.7 and 0.95 . Since PAR occurs occasionally, it is clear that the probability of performing PAR operation will be quite low. Usually, the PAR rate lies between 0.10 and 0.30 . Values in these ranges help the algorithm to strike a balance between exploration and exploitation.

The objective function $\left(\mathrm{Obj}_{\text {Fun }}\right)$ is the most important function for the optimization algorithms, where the best $\mathrm{Obj}$ Fun is proved the effectiveness of the optimization algorithm. The Obj $\mathrm{Fun}_{\text {Fun }}$ can be used to handle each generated solution vector which includes a group of sensor node positions. For the present problem, we have to maximize the coverage ratio of the network and minimize the number of added mobile nodes to the network, which is defined by the objective function. In other words, it is employed to scale the fittest of the positions and number of sensor node deployment. Each sensor node position is presented in real number and occurs at $x$ th row and $y_{\text {th }}$ column. Therefore, to maximize the coverage and minimize the number of sensors deployment, the objective function is calculated based on network coverage ratio $\left(C_{\text {ratio }}\right)$, HEWSN cost, and the minimum distance (MD). The objective function is depicted in the following equation.

$$
\mathrm{Obj}_{\mathrm{Fun}}=\left(\frac{1}{\text { SensCost }}\right) \times C_{\text {ratio }} \times \mathrm{MD}
$$

where the SensCost is defined as the cost of the number of sensor deployment and is calculated based on the following equation.

$$
\text { SensCost }=\frac{\left(\mathrm{HES}_{\mathrm{no}}-\mathrm{Min}_{\mathrm{noS}}\right)}{\operatorname{Max}_{\mathrm{noS}}-\operatorname{Min}_{\mathrm{noS}}}
$$

where $\mathrm{HES}_{\mathrm{no}}$ is the number of heterogeneous sensor deployment, and the aim is to be minimized, whereas Max _no ${ }_{S}$ and Min _no ${ }_{S}$ are calculated using Equations (10) and (11).

The coverage ratio $\left(C_{\text {ratio }}\right)$ is used to scale the QoS in the $\mathrm{Obj}_{\text {Fun }}$ of the proposed algorithm, where the aim is to be maximized. $C_{\text {ratio }}$ is computed by the following equation [57]:

$$
C_{\text {ratio }}=\frac{N_{E f f}}{\left(\text { Cells }_{W_{\text {Dim }}} \times \text { Cells }_{H_{\text {Dim }}}\right)},
$$

where $N_{E f f}$ is the number of positions that are covered adequately and is calculated based on $\operatorname{Probs}_{\mathrm{ov}}(\mathrm{t})$ value in Equation (16), whereas (Cells $\mathrm{W}_{\text {Dim }} \times$ Cells $_{\mathrm{H}_{\text {Dim }}}$ ) is the dimensions of whole AoI that was calculated in Equations (3) and (4).

$\mathrm{MD}$ is the minimum distance between sensors, where the aim is to prohibit the unwanted convergence between sensors that may happen through the construction procedure of the proposed method, and it is computed in the following equation.

$$
\mathrm{MD}=\frac{\operatorname{MD}\left(s_{\mathrm{i}}, \mathrm{s}_{\mathrm{j}}\right)}{\operatorname{MAX}(\text { Diagonal })}
$$

where the numerator is used to compute the minimum distance between sensors and is calculated in the following equation.

$$
\operatorname{MD}(\mathrm{Si}, \mathrm{Sj})=S_{i}^{n}\left(S_{j}^{n}\left(\sqrt{\left(x s_{i}-x s_{j}\right)^{2}+\left(y s_{i}-y s_{j}\right)^{2}}\right),\right.
$$

where $S_{i}^{n}$ and $S_{j}^{n}$ are the permutations of sensing range types for the deployment sensors. $x s_{i}$ and $y s_{i}$ are $x$-axis and $y$-axis of each sensor, respectively. For example, if any study has two types of sensors with different sensing ranges, the probabilities of MD will be calculated exponentially such as $2^{n}$. So, the complexity will be increased with increasing types of sensors with different sensing ranges.

The diagonal means the length of diagonal AoI. So, the divisor of $\mathrm{Obj}_{\text {Fun }}$ in Equation (20) is used to compute the diagonal for each type and then selects the largest one to reach the minimum value of $\mathrm{MD}$. The diagonal for each type is calculated in the following equation. 


$$
\text { Diagonal }=\sqrt{\left(W-R_{s_{i}}-U_{e}\right)^{2}+\left(H-R_{s_{i}}-U_{e}\right)^{2}},
$$

where $\boldsymbol{W}$ and $\boldsymbol{H}$ are the width and height of the AoI, whereas $\boldsymbol{R}_{\boldsymbol{s}_{i}}$ and $U_{e}$ are calculated in Equations (1) and (2), respectively.

3.3.2. Initialize the Harmony Memory. Initialize the HM matrix $(N \times \mathrm{HMS})$, where $N$ is the number of decision variables, and $M$ is HMS. Then fill the HM randomly by generating the feasible solution vectors. For each decision variable $\left(s_{i}\right)$, lower ${ }_{\mathrm{si}} \leq \mathrm{si} \leq$ upper $_{\mathrm{si}}$, where lower ${ }_{\mathrm{si}}$ and upper $_{\mathrm{si}}$ represent the lower and upper boundaries of allowed values, respectively. Formally, HM and the corresponding fitness function values are shown as follows:

$$
\mathrm{HM}=\left[\begin{array}{ccccc}
x_{1}^{1} & x_{2}^{1} & \cdots & x_{N-1}^{1} & x_{N}^{1} \\
x_{1}^{2} & x_{2}^{2} & \cdots & x_{N-1}^{2} & x_{N}^{2} \\
\vdots & \cdots & \cdots & \cdots & \cdots \\
x_{1}^{\mathrm{HMS}-1} & x_{2}^{\mathrm{HMS}-1} & \cdots & x_{N-1}^{\mathrm{HMS}-1} & x_{N}^{\mathrm{HMS}-1} \\
x_{1}^{\mathrm{HMS}} & x_{2}^{\mathrm{HMS}} & \cdots & x_{N-1}^{\mathrm{HMS}} & x_{N}^{\mathrm{HMS}}
\end{array}\right] \Rightarrow \begin{array}{cc}
\Rightarrow & f\left(X^{1}\right), \\
& \Rightarrow f\left(X^{\mathrm{HMS}-1}\right),
\end{array}
$$

where $\left[x_{1}^{i} x_{2}^{i} \cdots x_{N-1}^{i} x_{N}^{i}\right]$ represents the feasible solution vector $X^{i}$ in the harmony memory, and $f\left(X^{i}\right)$ represents its corresponding objective function. The width $(N)$ in Equation (23) of each solution vector can be modified and allowed each solution vector to encode a various number of sensors. The modifying in length grants the ability of HSA to select the optimal number of sensors as well as its optimal positions. Thus, to achieve this ability, the proposed work needs calculating range depending on the given network to avoid the extra computational demands and the false variable decisions on the number of nodes as mentioned in Equations (10) and (11). In addition, the length of each harmony memory vector represents as $\mathrm{HMV}_{\text {length}}$, will vary between $\mathrm{Min} \_\mathrm{no}_{s}$ and Max _no $o_{s}$ parameters, and is computed by the following equation.

$$
\mathrm{HMV}_{\text {length }}=\mathrm{Min} \_\mathrm{no}_{s}+\left(\operatorname{Max}_{\text {nos }}-\operatorname{Min}_{\text {nos }}\right) \times \operatorname{rand}()
$$

where Min _no ${ }_{s}$ and $\mathrm{Max} \_\mathrm{no}_{s}$ are calculated as Equations (10) and (11), respectively. The following example explains the role of $\mathrm{HMV}_{\text {length }}$ on the enhancement of the network coverage and minimizing the cost of the network in heterogeneous case.

Example 1. Assume existence AoI with the size of $\left(50 \times 50 \mathrm{~m}^{2}\right)$ and many requirements such as finding the number of the optimal sensor with optimal positions. For example, two types of sensor nodes have various sensing range $\left(R_{s_{i}}\right) 10$ and 5 meters, respectively. Uncertainty values of $U_{e}$ are assumed to be half of $R_{s_{i}}$ for each one. Therefore, Min _no ${ }_{s}$ is computed in Equation (10) and equal to 3 and 11 sensors, respectively, while $\mathrm{Max}_{-} \mathrm{no}_{s}$ is calculated in Equation (11) and equal to 25 and 100 sensors, respectively. Thus, the $\mathrm{HMV}_{\text {length }}$ is mod- ifying between ( 3 and 100) sensors. Assume one of the harmony memory vectors that have 5 node positions and monitored by 5 sensor nodes:

\#; \#; 2.4; 5.8 \#; \#; 12.5; 16.8; \#; \#; 33.2; 3.4; 15.7; 44.8; \#; \#; $\# ; \# ; 8 ; 7.2 ;:::$

In the previous vector, the $x$-axis and $y$-axis of the first node are (2.4 and 5.8), the positions of the second node are (12.5 and 16.8), the positions of the third node are (33.2 and 3.4), the positions of the fourth node are (15.7 and 44.8 ), and finally, the positions of the fifth node are ( 8 and 7.2). The positions which signed with "\#" are unused positions and are encoded to make the length of the $\mathrm{HMV}_{\text {length }}$ to be as the Max _no ${ }_{s}$. In the end, every harmony memory vector is generated with a various number of candidate sensor node positions. Hence, the proposed $\mathrm{Obj}_{\mathrm{Fun}}$ will measure and compute the quality of each harmony memory vector as the quality of the network coverage and cost as defined in step 1. The unused factor "\#” that may exist in the harmony memory vector is deleted, and the rest of the components are employed to cover the AoI.

\subsubsection{Improvise a New Harmony. A new Harmony} vector $X^{\prime}=\left(x_{1}^{\prime} x_{2}^{\prime} \cdots x_{N}^{\prime}\right)$ is generated based on three parameters: memory consideration, pitch adjustment, and random selection which are modified to matching the WSN nature as follows.

For each component $x_{i}^{\prime}$, pick up the corresponding component of $\mathrm{x}_{i}^{j}$ randomly from any of the values in the specified HM range $\left(x_{i}^{1}-x_{i}^{\mathrm{HMS}}\right)$ with the probability of $P_{\mathrm{hmcr}}$.

$$
x_{i}^{\prime} \leftarrow\left\{\begin{array}{cr}
x_{i}^{\prime} \in\left\{x^{\prime}=\left(x_{i}^{1}, x_{i}^{2}, \cdots: x_{i}^{\mathrm{HMS}}\right.\right. & \text { with prob.HMCR } \\
x_{i}^{\prime} \in X i & \text { with prob.(1- HMCR })
\end{array}\right\} .
$$

For example, the values of the $x$-axis $\left(x_{\mathrm{si}}{ }^{1}, x_{\mathrm{si}}{ }^{2}, x_{\mathrm{si}}{ }^{3}, \cdots\right.$, $\left.x_{\mathrm{si}}{ }^{\mathrm{HMS}}\right)$ and the $y$-axis $\left(y_{\mathrm{si}}{ }^{1}, y_{\mathrm{si}}{ }^{2}, y_{\mathrm{si}}{ }^{3}, \cdots, y_{\mathrm{si}}{ }^{\mathrm{HMS}}\right)$ are selected from HM randomly and proceed in a similar way for the remnant components. On the other hand, the values can be picked randomly from the range of allowed values with the probability of $1-P_{\mathrm{hmcr}}$, where $\mathrm{xs}_{i} \in\left[\right.$ lower $_{x_{s}}$; upper $\left._{x_{s}}\right]$ and $y s_{i} \in\left[\right.$ lower $_{y_{s}} ;$ upper $\left._{y_{s}}\right]$. For example, HMCR of 0.95 indicates that the probability of the HS algorithm to choose the decision variable values from historically stored values in the $\mathrm{HM}$ is $95 \%$, and the probability of selecting a new random value from the allowed range is (100-95)\%.

After the harmony memory considering factor is completed, change $x_{i}^{\prime}$ with the probability of $P_{\text {par }}$. The pitch adjustment is applied only if the value is chosen from the HM. The pitch adjustment procedure is illustrated in the following equation.

$$
\text { Pitch adj. dec.for } x_{i}^{\prime} \leftarrow\left\{\begin{array}{c}
\text { Yes with prob.PAR, } \\
\text { No with prob.(1-PAR). }
\end{array}\right\},
$$


TABLE 1: Simulation parameters.

\begin{tabular}{|c|c|c|}
\hline Parameter & & Value \\
\hline \multirow{2}{*}{ Area of interest (AoI) } & AoI 1 & $50 \times 50 \mathrm{~m}^{2}$ \\
\hline & AoI 2 & $100 \times 100 \mathrm{~m}^{2}$ \\
\hline Size of each cell & & $10 \mathrm{~m}$ \\
\hline Maximum iterations number (MItr) & & 60000 iterations \\
\hline \multirow{2}{*}{ Experiments number } & AoI 1 & 12 runs of each scenario \\
\hline & AoI 2 & 10 runs of each scenario \\
\hline Coverage threshold $\left(C_{\mathrm{th}}\right)$ & & 0.9 \\
\hline \multirow{4}{*}{ Parameters describing the sensing nodes. } & $\alpha_{1}$ & 1 \\
\hline & $\alpha_{2}$ & 0 \\
\hline & $\beta_{1}$ & 1 \\
\hline & $\beta_{2}$ & 0.5 \\
\hline Uncertainty range $\left(U_{e}\right)$ & & 5 and 2.5 \\
\hline \multirow{2}{*}{ Harmony memory size (HMS) } & AoI 1 & 30 vectors \\
\hline & AoI 2 & 50 vectors \\
\hline Harmony memory consideration rate (HMCR) & & $90 \%$ \\
\hline Pitch adjustment rate (PAR) & & 0.3 \\
\hline Bandwidth distance $\left(\mathrm{BW}_{\text {range }}\right)$ & & 0.2 \\
\hline Runs & & Over 30 runs \\
\hline
\end{tabular}

If the pitch adjustment decision for $x_{i}^{\prime}$ is yes, then the small amount bw of changes takes place for pitch adjustments:

$$
x_{i}^{\prime} \leftarrow x_{i}^{\prime} \pm \mathrm{BW}_{\text {range }} * \text { rand }()
$$

where the parameter $\mathrm{BW}_{\text {range }}$ is the distance bandwidth which is used to modify the new node position value. The change is applied for the values of the $x$-axis and the $y$-axis of the corresponding $x_{i}^{\prime}$.

3.3.4. Harmony Memory Update. Evaluate the new harmony $x^{\prime}=\left(x_{1}^{\prime} x_{2}^{\prime} \cdots x_{N}^{\prime}\right)$ by calculating its objective function $\mathrm{Obj}_{\mathrm{Fun}}$ using Equation (23). If the value of its $\mathrm{Obj}_{\text {Fun }}$ is better than that of the worst harmony $x^{\text {worst }}$ in the HM, $x^{\prime}$ is included in the HM, and the existing $x^{\text {worst }}$ is excluded from the HM. Subsequently, the vectors are sorted based-on their $\mathrm{Obj}_{\text {Fun }}$ values.

3.3.5. Termination Criterion Check. In this step, repeat steps 3 at Subsections 3.3.3 and 4 at Subsection 3.3.4 until the termination criterion is met. The solution vector in HM that can achieve maximizing coverage and minimizing the number of sensors deployment is selected to be the best solution vector.

\section{Experimental Results and Comparative Study}

4.1. Simulation Setup. The simulation environment is based on PSM and HEWSN random deployment using HSA. The simulations were applied over two different areas in three scenarios for each one. In the first area, the AoI is designed to be $50 \times 50 \mathrm{~m}^{2}$ dimension area which is separated into 25 cells and all results based on 25 sensors as a maximum number of sensor deployment. In the second area, the AoI is designed to be $100 \times 100 \mathrm{~m}^{2}$ dimension area which is separated into 100 cells and all results based on 50 sensors as a maximum number of sensors deployment. Most of the simulation parameters in Table 1 are set according to [57]. The measure of cell in each scenario is set to be $10 \mathrm{~m}$, as mentioned in Figure 3. The sensing range $R_{s}$ has two types $A$ and $B$, where type $A$ is set to be $10 \mathrm{~m}$ and type $B$ set to be $5 \mathrm{~m}$. The uncertainty in sensor detection $R_{e}$ is set to be half of the sensing range $R_{s}$ (means 5 and 2.5, respectively). The coverage threshold $C_{\text {th }}$ is set to be 0.9 , whereas the PSM's parameters are set to be $\alpha_{1}=1 ; \alpha_{2}=0 ; \beta_{1}=1$, and $\beta_{2}=0.5$.

The values of $\lambda_{1}$ and $\lambda_{2}$ are calculated based on Equations (14) and (15). In addition, the minimum and maximum numbers of sensors that achieved the network requirements are calculated according to Equations (10) and (11), respectively. Finally, the simulation worked on a computer that has windows 7, core i5-3470, 8 GB RAM, and CPU 3.20 GHz. More details about simulation environment parameters are illustrated in Table 1.

The HMCR and PAR are two fundamental parameters in HSA. They are used to set the probability of components stored in HM. If the value of HMCR is extremely high (near 1), almost all the harmonies are used in the HM. Therefore, typically, we used $\mathrm{HMCR}=90 \%$ and the HMS $=30$ solution vectors as the work in [11].

On the other hand, a low PAR with a little value of bandwidth can slow down the convergence of HSA. However, a very high PAR with a high-value bandwidth may also lead to wrong solutions [64]. Thus, the applications usually 
TABLE 2: Results of HEWSN deployment based on HSA.

\begin{tabular}{lccc}
\hline Cell size vs. sensing range & \multicolumn{2}{c}{ HEWSN deployment 10(10,5) } & S2 \\
\hline Number of runs & 12 & 12 & 12 \\
Maximum number of node sensors (MN) & 25 & 25 & 25 \\
Best (maximum coverage ratio) & $96 \%$ & $100 \%$ & $100 \%$ \\
Number of corresponding sensors & 16HES $=(12 \mathrm{HOS})$ & $18 \mathrm{HES}=(13.5 \mathrm{HOS})$ & $17 \mathrm{HES}=(12.5 \mathrm{HOS})$ \\
Corresponding sensor ratio & $48 \%$ & $54 \%$ & $50 \%$ \\
Average of each scenario & $88 \%$ & $92 \%$ & $94 \%$ \\
\hline
\end{tabular}

TABLE 3: The best minimum cost of HEWSN deployment based on HSA.

\begin{tabular}{lc}
\hline Cell size versus sensing range & $\begin{array}{c}\text { HEWSN deployment } \\
10(10,5)\end{array}$ \\
\hline Maximum number of node sensors & 25 \\
Number of corresponding sensors & $17 \mathrm{HES}=12.5 \mathrm{HOS}$ \\
Best (maximum coverage ratio) & $100 \%$ \\
Corresponding sensor ratio & $50 \%$ \\
Cost & $50 \%$ \\
\hline
\end{tabular}

TABLE 4: The best result of HEWSN deployment over $100 \times 100 \mathrm{~m}^{2}$.

\begin{tabular}{lccc}
\hline Cell size versus sensing range & \multicolumn{3}{c}{$10(10,5)$} \\
Scenarios of AoI 2 & S1 & S2 & S3 \\
\hline Maximum number of node sensors (MN) & 50 & 50 & 50 \\
Number of runs & 10 & 10 & 10 \\
Number of corresponding sensors & 42 & 45 & 49 \\
Best (maximum coverage ratio) & $79 \%$ & $88 \%$ & $90 \%$ \\
Corresponding sensor ratio & $64 \%$ & $67 \%$ & $73 \%$ \\
Average of each scenario & $69 \%$ & $76 \%$ & $82 \%$ \\
Cost & $15 \%$ & $21 \%$ & $17 \%$ \\
\hline
\end{tabular}

use $\mathrm{PAR}=0.1$ to 0.5 . $\mathrm{PAR}$ value $=0.3$ which means that the neighboring value will be chosen with a $30 \%$ probability [11].

The proposed model is benchmarked against the baseline cases of sensor network deployment [11]. Several metrics are selected to evaluate the efficiency and effectiveness of the proposed model named coverage ratio, the minimum number of sensors deployment (network cost), and reducing the overlap among sensor nodes. The parameters that are mentioned in Tables $2-5$ such as corresponding sensors ratio $\left(\right.$ Corr $\left._{\text {ratio }}\right)$ and cost are calculated in Equations (28) and (29).

The coverage ratio $\left(C_{\text {ratio }}\right)$ was given in Equation (19), and the corresponding sensors ratio ( Corr $_{\text {ratio }}$ ) is calculated by Equation (28) and depicted in Equation (29).

$$
\text { Corr }_{\text {ratio }}=(\text { corresp. No. of sensors } \div \mathrm{MN}) \times 100 \text {, }
$$

where the corresponding number of sensors means the number of deploying sensors, whereas $\mathrm{MN}$ means the maximum number of deploying sensors.
The minimum number of deploying sensors (network cost) is calculated based on Equation (28).

$$
\text { Cost }=C_{\text {ratio }}-\text { Corr }_{\text {ratio }} \text {. }
$$

4.2. Simulation Results. Three simulation scenarios have been created to show the performance of the proposed model in both AoI of size $50 \times 50 \mathrm{~m}^{2}$ and AoI of size $100 \times 100 \mathrm{~m}^{2}$. For comparison purposes, the proposed model in HEWSN deployment has been simulated, and the simulation results of HSA in HOWSN deployment have been inserted. The performance of the proposed model has been evaluated in three metrics: maximizing coverage, minimizing network cost, and reducing the overlap of sensor nodes using MATLAB simulator. The three scenarios of the proposed model are explained in the following subsections.

4.2.1. Scenarios of the Proposed Model over AoI 1. The proposed model conducted three scenarios to confirm the efficiency and effectiveness of the proposed deployment model for solving the network coverage and cost problems in WSN. Each scenario executes 12 runs, as presented in Table 2. One best result of each scenario was chosen depending on the best minimum number of HEWSN random deployment to offer an efficient solution for coverage problem as follows.

(1) Maximizing Coverage of HEWSN Based on HSA. The first part of the main problem which is investigated is to maximize the coverage. Each scenario used 25 sensors as the maximum number of deployed sensors. In the best result of the first scenario, the corresponding number of deploying sensors is 16 out of 25 sensors ( 8 sensors with sensing range $5 \mathrm{~m}$ and 8 sensors with sensing range $10 \mathrm{~m}$ ) as illustrated in Figure 7 . In the best result of the second scenario, the proposed model used less number of sensors, where the corresponding number of deploying sensors is 18 out of 25 sensors ( 9 sensors with sensing range $5 \mathrm{~m}$ and 9 sensors with sensing range $10 \mathrm{~m}$ ) as illustrated in Figure 8. Finally, in the best result of the third scenario, the best corresponding number of deploying sensors is 17 out of 25 sensors ( 9 sensors with sensing range $5 \mathrm{~m}$ and 8 sensors with sensing range $10 \mathrm{~m}$ ) as illustrated in Figure 9.

It is clear from the results shown in Figures 7-9, the coverage ratio of HSA increases with the decreased number of sensors. The first best result that is obtained in Figure 7 
TABLE 5: Results of HEWSN deployment based on GA.

\begin{tabular}{|c|c|c|c|c|c|}
\hline \multirow{2}{*}{ Cell size versus sensing range } & \multicolumn{5}{|c|}{ HEWSN deployment $10(10,5)$} \\
\hline & S1 & S2 & S3 & S4 & S5 \\
\hline $\begin{array}{l}\text { Maximum number of node sensors } \\
\text { (MN) }\end{array}$ & 25 & 25 & 25 & 30 & 35 \\
\hline Number of runs & 12 & 12 & 12 & 12 & 12 \\
\hline Number of corresponding sensors & 20HES $=15$ HOS & $22 \mathrm{HES}=16.5 \mathrm{HOS}$ & 23HES $=17$ HOS & $27 \mathrm{HES}=20.5 \mathrm{HOS}$ & $34 \mathrm{HES}=23.5 \mathrm{HOS}$ \\
\hline Best (maximum coverage ratio) & $56 \%$ & $60 \%$ & $60 \%$ & $68 \%$ & $84 \%$ \\
\hline Corresponding sensor ratio & $60 \%$ & $66 \%$ & $68 \%$ & $58 \%$ & $61 \%$ \\
\hline Average of each scenario & $44 \%$ & $50 \%$ & $55 \%$ & $60 \%$ & $67 \%$ \\
\hline
\end{tabular}

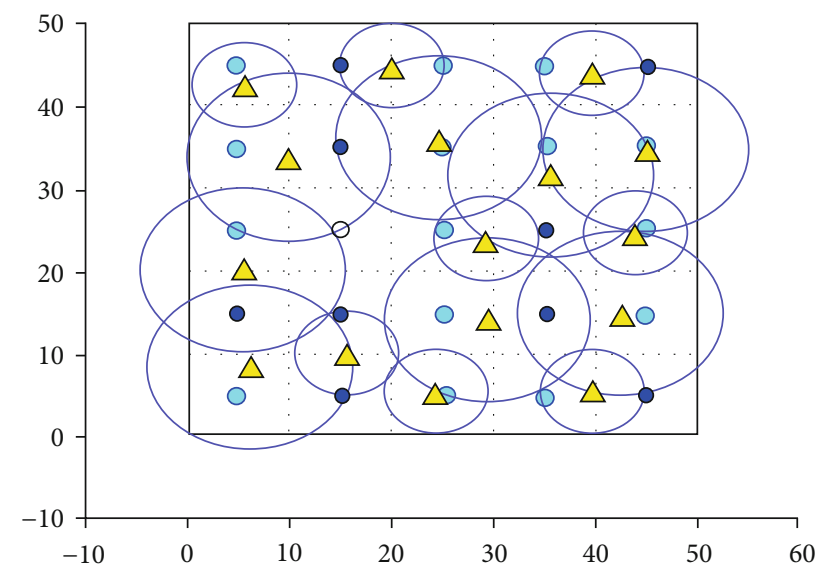

FIGURE 7: Best result of first scenario in HEWSN deployment with $10 \mathrm{~m}$ and $5 \mathrm{~m}$ using $16 \mathrm{HES}=12 \mathrm{HOS}$ sensors based on HSA.

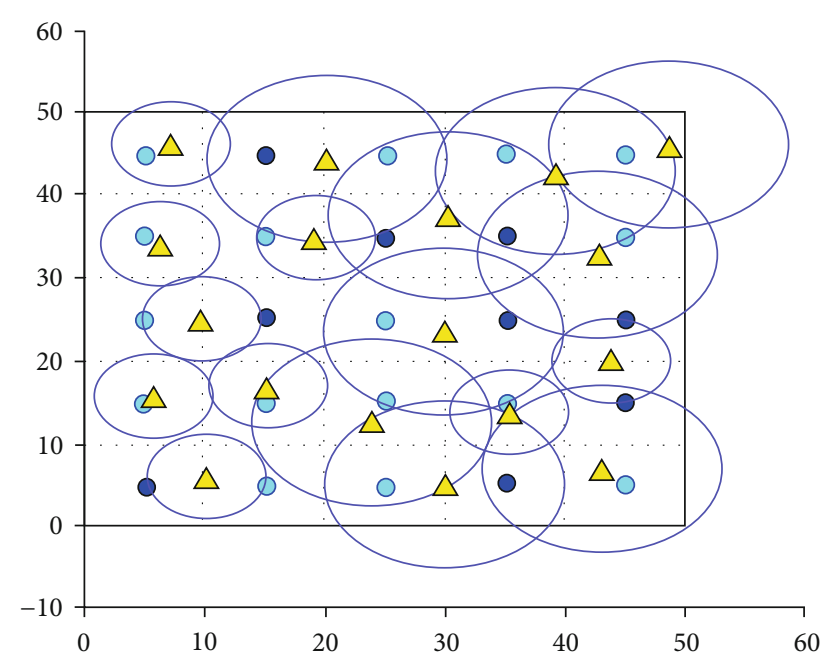

Figure 8: Best result of the second scenario in HEWSN deployment with $10 \mathrm{~m}$ and $5 \mathrm{~m}$ using $18 \mathrm{HES}=13.5 \mathrm{HOS}$ sensors based on HSA.

shows that the coverage of the proposed model was near the full coverage, where the coverage ratio reached at $96 \%$ when the corresponding number of deploying sensors is 16 sensors and the corresponding sensor ratio is $48 \%$. In the second result, the coverage ratio reached $100 \%$ and $54 \%$ of the corresponding sensor ratio when the corresponding number of

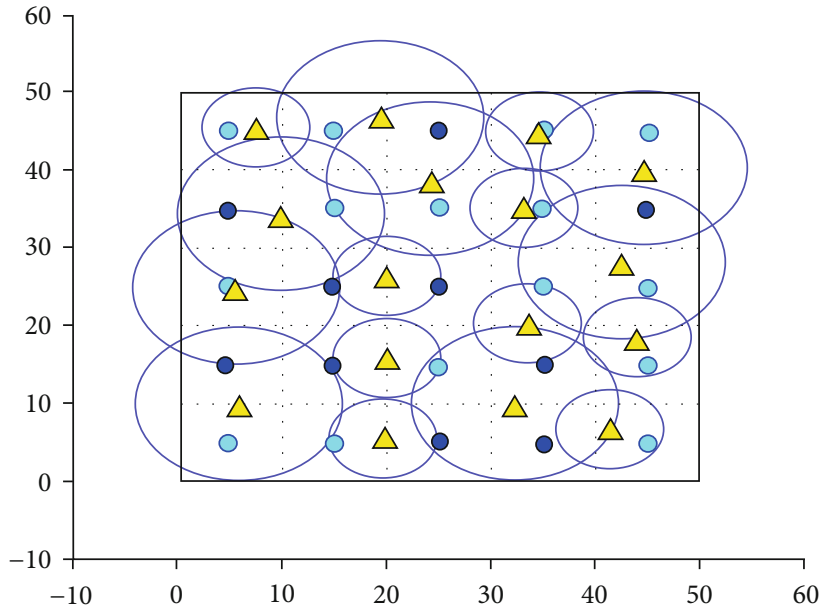

FIGURE 9: Best result of the third scenario in HEWSN deployment with $10 \mathrm{~m}$ and $5 \mathrm{~m}$ using $17 \mathrm{HES}=12.5 \mathrm{HOS}$ sensors based on HSA.

deploying sensors is 18 sensors, as shown in Figure 8 . The third result in Figure 9 shows the best performance of the proposed model that has an ability to cover all of the targets and find the optimal positions of the deploying sensor nodes with a minimum number of sensors. Table 2 summarized the best results of the three scenarios based on the proposed model. The average coverage ratio for 12 runs in each scenario was $88 \%, 92 \%$, and $94 \%$.

(2) Minimizing Cost of HEWSN Based on HSA. The second part of the main problem, which is also investigated to minimize the network cost. The number of cells in the AoI 1 is equal to the maximum number of deployed sensors. Table 3 shows a summary of HEWSN with the best minimum cost based HSA as follows.

As shown in Table 3, the cost of the best result of HEWSN deployment was reduced by $50 \%$ when the number of corresponding sensors is equal to 12.5 HOS out of 25 sensors.

4.2.2. Scenarios of the Proposed Model over AoI 2. In this scenario, the proposed model was investigated to offer an efficient solution for coverage and cost problems in a broad area. The cost consideration for the number of sensors deployment in a small area can be determined, but to cover 


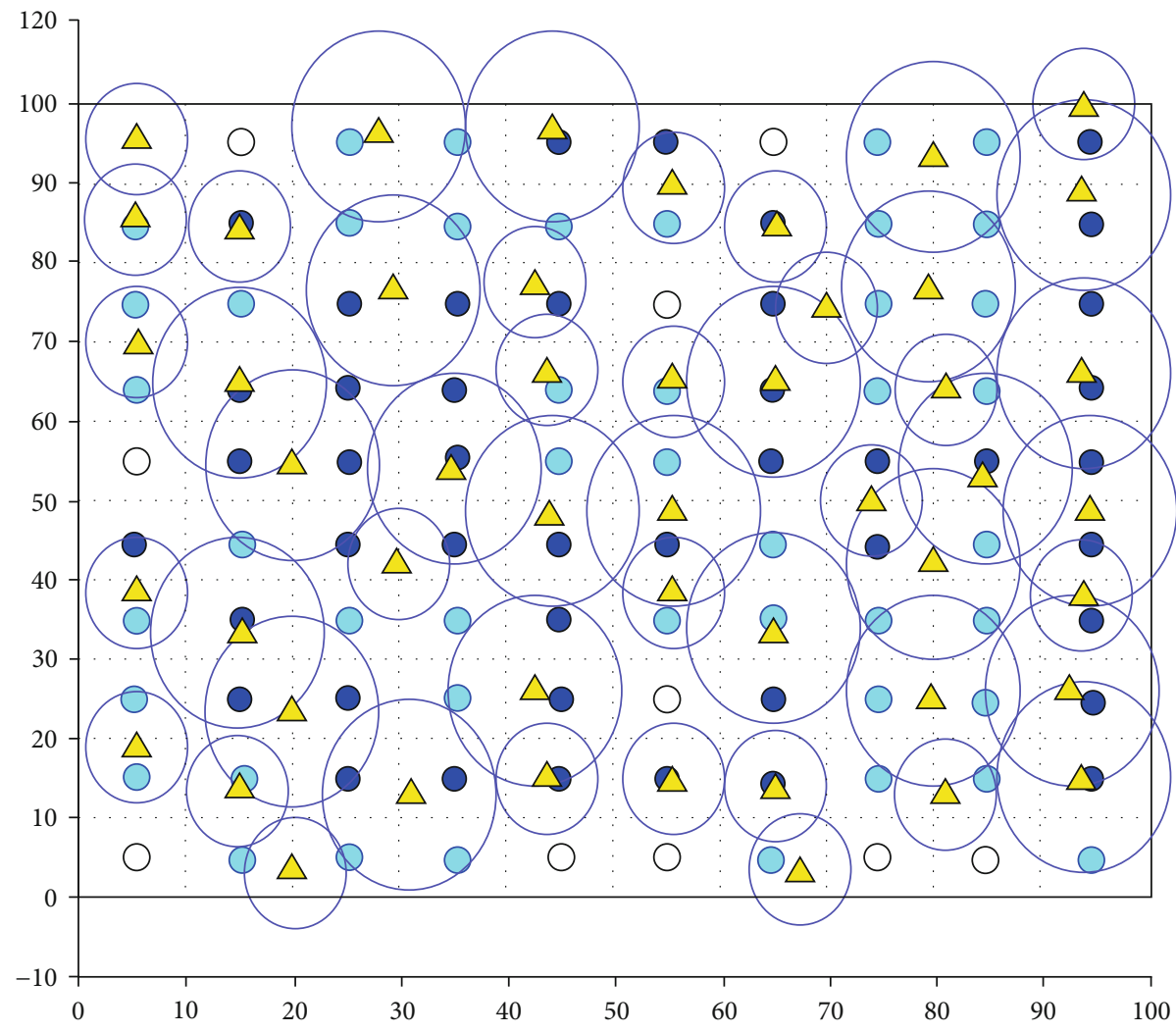

Figure 10: Best result of AoI $100 \times 100 \mathrm{~m}^{2}$ based on HSA.

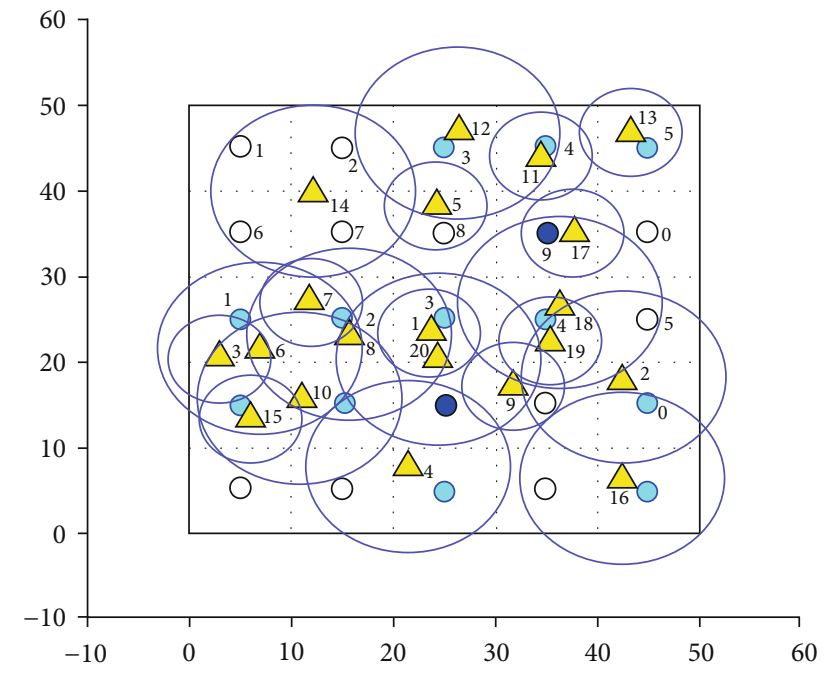

FIGURE 11: Best result of first scenario in HEWSN deployment with $10 \mathrm{~m}$ and $5 \mathrm{~m}$ using $20 \mathrm{HES}=15 \mathrm{HOS}$ sensors based on GA.

the broad area using sensor networks is still necessary and undetermined. Three scenarios were considered to optimize the proposed model performance in terms of coverage ratio and minimum cost over a large scale. These scenarios are aimed at knowing the ability of the proposed model on both maximizing the coverage and minimizing the number of deployed sensors. Table 4 summarized the three scenarios, and Figure 10 depicts the best result of AoI 2 as follows.

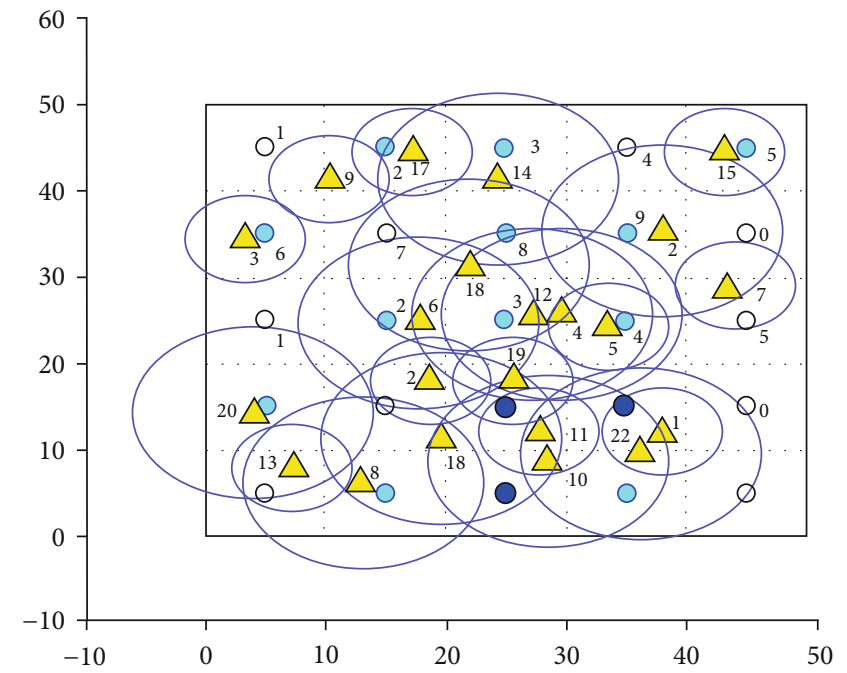

Figure 12: Best result of the second scenario in HEWSN deployment with $10 \mathrm{~m}$ and $5 \mathrm{~m}$ using $22 \mathrm{HES}=16.5 \mathrm{HOS}$ sensors based on GA.

As shown in Table 4, the coverage ratio reached at $90 \%$ when the number of deploying sensors equal to 49 out of 50 sensors. The average of the best scenario was $82 \%$, and the cost was reduced by $17 \%$.

4.3. Comparative Study. This section presents two comparisons, the first comparison between the proposed model and GA in HEWSN and the second comparison between HSA 


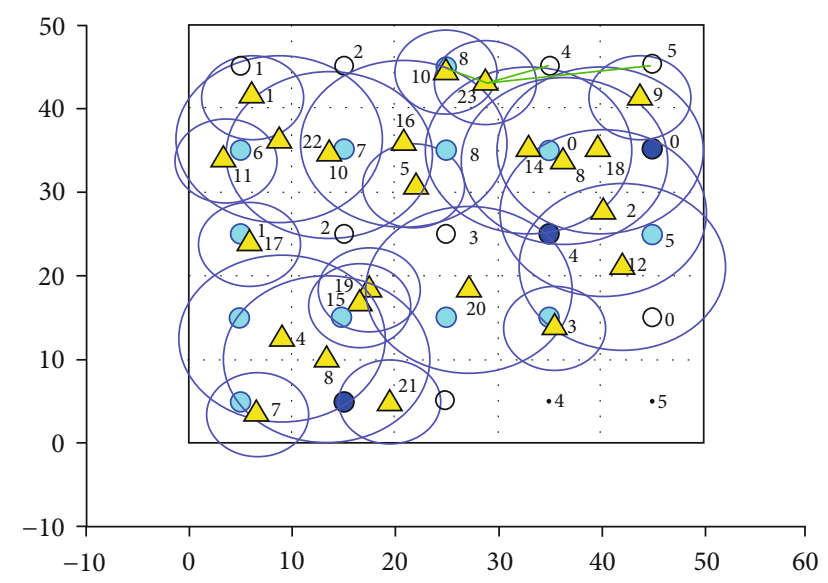

FIGURE 13: Best result of the third scenario in HEWSN deployment with $10 \mathrm{~m}$ and $5 \mathrm{~m}$ using $23 \mathrm{HES}=17 \mathrm{HOS}$ sensors based on GA.

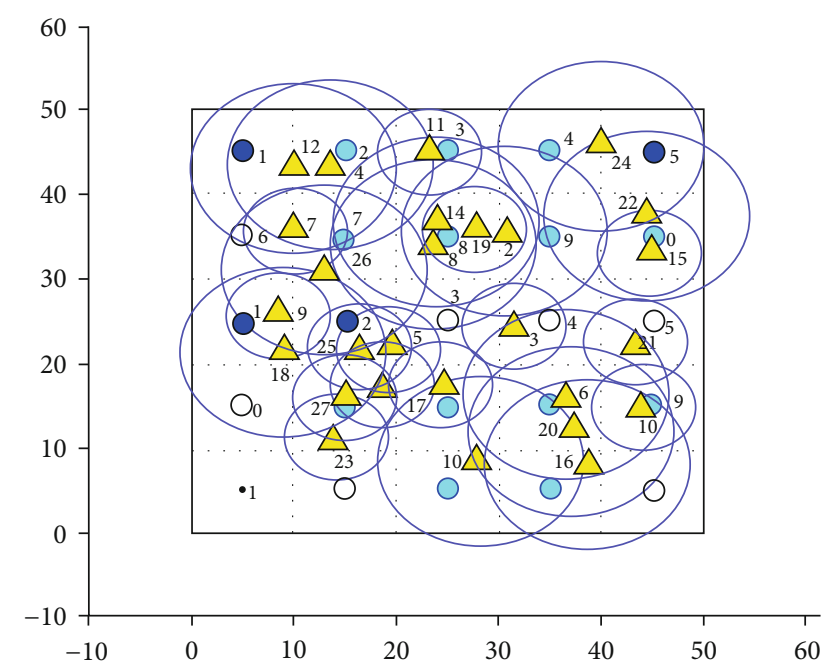

FIgURE 14: Best result of $4^{\text {th }}$ scenario using 27 sensors deployment based on GA.

and GA in HOWSN. Both comparisons have been evaluated into two metrics, maximizing coverage and minimizing cost as follows.

4.3.1. Scenarios of Genetic Algorithm (GA). In Genetic Algorithm, the group of solutions is known as chromosomes. These chromosomes represent a group of sensor node positions and are stored in population, which is equivalent to Harmony Memory in Harmony Search. In each generation of GA, a set of population size chromosomes is known as offsprings. In our scenarios, a population size is equal to 50 , GA terminates after 1,000 generations, and a single point crossover operator is used with a probability of $\mathrm{Pc}=(0.80)$.

For the mutation operator, if a generated random number is with a probability of $\mathrm{Pm}=(0.025)$, each gene in the offspring can be modified using Equation (27). The values of both $\mathrm{Pm}$ and $\mathrm{Pc}$ are taken according to the comparison application in [11].

In the previous section, the simulation results are executed to investigate the performance of the proposed

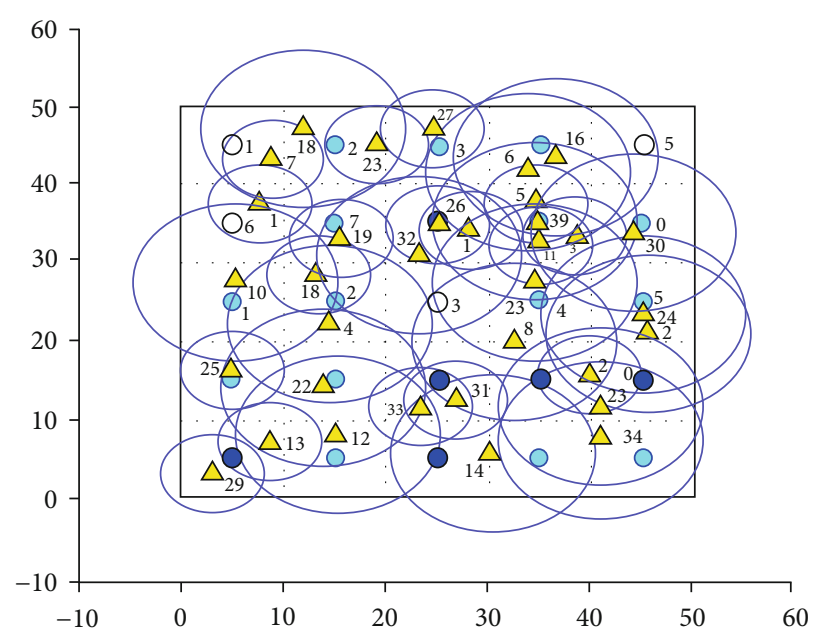

FIGURE 15: Best result of $5^{\text {th }}$ scenario using 34 sensors deployment based on GA.

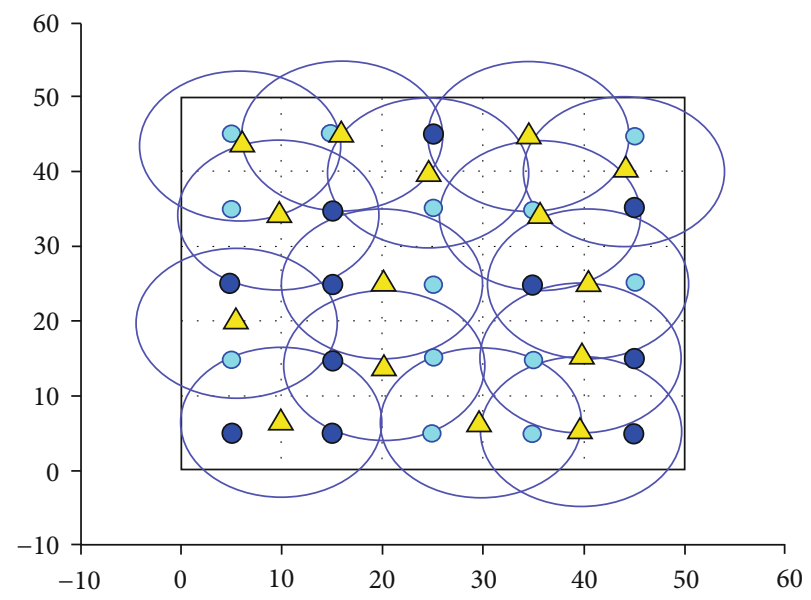

FIgURE 16: Best result of HOWSN deployment with $10 \mathrm{~m}$ using 15 sensors based on HSA.

model. In this section, three scenarios are introduced in this simulation using 12 runs. One best result of each scenario is chosen depending on the best minimum number of HEWSN random deployment as illustrated in Figures 1113. In the best result of the first scenario, the corresponding number of deploying sensors is 20 out of 25 sensors ( 10 sensors with sensing range $10 \mathrm{~m}$ and ten sensors with sensing range $5 \mathrm{~m}$ ) as illustrated in Figure 11. In the best result of the second scenario, the corresponding number of deploying sensors is 22 out of 25 sensors (11 sensors with sensing range $5 \mathrm{~m}$ and 11 sensors with sensing range $10 \mathrm{~m}$ ) as illustrated in Figure 12. In the best result of the third scenario, the corresponding number of deploying sensors is 23 out of 25 sensors (12 sensors with sensing range $5 \mathrm{~m}$ and 11 sensors with sensing range $10 \mathrm{~m}$ ) as illustrated in Figure 13.

As shown in Figure 11, the coverage ratio reached at 56\% when the corresponding number of deploying sensors equal to 20 sensors, and the corresponding sensor ratio is $60 \%$. In the second and the third-best results, the coverage ratio reached 
TABLE 6: Best result comparison between HEWSN and HOWSN deployment based on HSA and GA.

\begin{tabular}{lccc}
\hline Parameters & HEWSN (HSA) & HEWSN (GA) & HOWSN (HSA) \\
\hline Number of runs & Over 30 & Over 30 & Over 30 \\
Cell size versus sensing range & $10(10,5)$ & $10(10,5)$ & $10(10)$ \\
Maximum number of sensors & 25 & 35 & 25 \\
Corresponding sensors & 12.5 & $\mathbf{3 4 H E S}=(\mathbf{2 3 . 5 H O S})$ & 15 \\
Best (maximum coverage ratio) & $100 \%$ & $84 \%$ & $\mathbf{1 0}$ \\
Corresponding sensor ratio & $50 \%$ & $61 \%$ & $60 \%$ \\
Cost minimization by & $50 \%$ & $23 \%$ & $40 \%$ \\
Average of (best scenario) & $94 \%$ & $67 \%$ & $93 \%$ \\
\hline
\end{tabular}

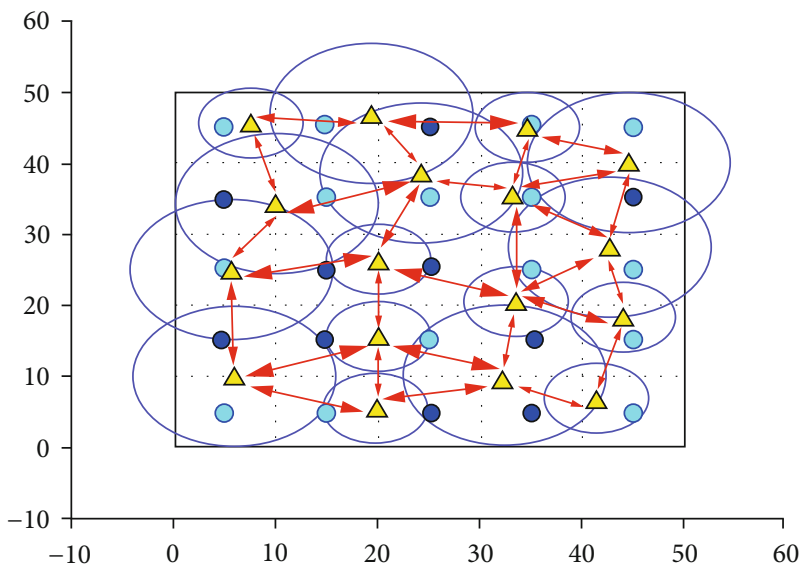

FIGURE 17: The impact of overlapping on the best result of the proposed model.

at $60 \%$ when the corresponding number of deploying sensors equal to 22 and 23 and the corresponding sensor ratio equal to $66 \%$ and $68 \%$ as shown in Figures 12 and 13, respectively.

Moreover, and to be more precise, GA dose did not reach maximum coverage ratio with a minimum number of sensors (15 of HOWSN or 17 of HEWSN), but it can be reached at $68 \%$ as the best result of the $4^{\text {th }}$ scenario when the corresponding number of deploying sensors equal to 27 and the maximum number of sensors deployment equal to 30 as shown in Figure 14. Also, it can be reached at $84 \%$ as the best result of the $5^{\text {th }}$ scenario when the corresponding number of deploying sensors equal to 34 sensors and the maximum number of sensor deployment equal to 35 as shown in Figure 15. Table 5 is summarized the best results of the five scenarios based on GA.

As shown in Table 5 , the best results of the $4^{\text {th }}$ and $5^{\text {th }}$ scenarios increased with the increasing number of deployed sensors. The coverage ratio of the $4^{\text {th }}$ scenario reached $68 \%$ when the number of deploying sensors equal to 27 . In addition, it reached $84 \%$, i.e., with increasing $16 \%$, when the number of deploying sensors identical to 34 sensors as in the $5^{\text {th }}$ scenario. The average coverage ratio of HEWSN random deployment in each scenario based on GA was $44 \%, 50 \%$, $55 \%, 60 \%$, and $67 \%$, respectively.

4.3.2. The Comparison of the Proposed Model with HSA in HOWSN. For comparison purpose, Figure 16 in [11] illus- trated the best simulation result of HOWSN deployment based on HSA.

As shown in Figure 16, the coverage ratio reached at $100 \%$ when the corresponding number of deploying sensors is 15 out of 25 sensors with a $60 \%$ corresponding sensor ratio. The cost of HOWSN deployment was reduced by $40 \%$, whereas the average coverage ratio was around $93 \%$ for all results.

Table 6 illustrates the comparison between the proposed model and both GA and HSA in HEWSN and HOWSN random deployment, respectively.

As shown in Table 6, the coverage ratio of HEWSN based GA increases with an increased number of corresponding deploying sensors. The coverage ratio reached $84 \%$ when the number of corresponding sensors was 34 sensors. In addition, the cost was reduced by $23 \%$. In other words, GA can be reached near full coverage when the number of deploying sensors was more than the number of cells.

In summary, the proposed model introduced the superiority against GA in HEWSN and increased by about $16 \%$ and $27 \%$ in terms of coverage ratio and cost, respectively. In addition, the cost of HOWSN based on HSA was reduced by $40 \%$, whereas the cost of HEWSN deployment was reduced by $50 \%$. Moreover, the coverage ratio of the proposed model was $94 \%$, whereas it was $93 \%$ in HOWSN based on HSA.

4.4. The Impact of Minimum Overlapping on the Proposed Deployment Model. The third metric is to minimize the overlapping coverage area in WSN with nodes having a different radius. This section illustrates the impact of the decreasing overlap between sensors that are introduced in the $\mathrm{Obj}_{\text {Fun }}$ of the proposed model. In fact, the MD operator is defined to prevent the deployed sensors from being close to each other and then avoid the network from overlapping problem, i.e., avoid an extra cost of the unnecessary deployed sensors. In other words, the coverage ratio is maximized by minimizing the overlap problem. It is clear that the overlap among sensors in Figure 9 was low. Each sensor is about the same distance from the other sensor.

In addition, Figure 9 can be able to obtain the best network coverage due to all target points were covered with a minimum network cost. On the other hand, Figure 15 has the least displacement among sensors, where the number of sensors that are used to cover the network is higher than that in Figure 9. It used 34 sensors close to each other and then the coverage was minimized by around $16 \%$ (the rest to reach 
maximum coverage 100\%). This means that (Figure 15) the number of demanded sensors to get maximum coverage is to be around 40 sensors. This extra 23 (the extra numbers to 17 sensors of best Figure 9) sensors can increase the network cost by around $42 \%$ compared to the best result in Figure 9. Figure 17 shows the impact of overlapping on the best result of the proposed model.

\section{Conclusion}

In this paper, we addressed the problem of maximizing the coverage and minimizing the number of deployment of sensors in HEWSN using HSA and PSM. One of the main contributions of our work is to update and reformulate the equations that are used in PSM for homogeneous wireless sensor network and to evaluate the proposed deployment model by comparison with the existing method based on HSA. The proposed work introduced heterogeneous sensor network deployment based on the probabilistic sensing model (PSM) and harmony search algorithm (HSA). PSM is used to enhance the probability of coverage without interference. Here, HSA is used for optimizing the maximum coverage with the minimum number of sensors in HEWSN. The simulation results and quantitative analysis proved that the proposed model can provide solutions up to $100 \%$ of the coverage ratio over a small area. Also, it demonstrated the efficiency and superiority of the proposed algorithm in HEWSN random deployment with the minimum number of sensor nodes, where it reduced the network deployment cost by a factor of $50 \%$ with a minimum overlapping.

On the other hand, the proposed work has evaluated the performance of the proposed algorithm with HSA in HOWSN random deployment. The results showed that the proposed model reduced the deployment cost by a factor of $10 \%$ by comparing with HSA in HOWSN deployment. Our contribution here is twofold. First, the minimum cost of heterogeneous sensors for PSM as the maximizing coverage in heterogeneous wireless sensor network (HEWSN) model has been reformulated, and second, using of HSA and PSM for providing optimum deployment in HEWSN.

\section{Data Availability}

The data (measured data and codes) used to support the findings of this study are available from the corresponding author upon request and with the institute's permission.

\section{Conflicts of Interest}

The author declares that they have no competing interests.

\section{Acknowledgments}

The authors would like to thank Dr. Osama Moh'd Alia for his quick responses and valuable guidance to accomplish this article research.

\section{References}

[1] L. Mainetti, L. Patrono, and A. Vilei, "Evolution of wireless sensor networks towards the internet of things: a survey," in 19th International Conference, Software, Telecommunications and Computer Networks (SoftCOM), Split, Croatia, 2011.

[2] O. Zorlu and O. K. Sahingoz, "Increasing the coverage of homogeneous wireless sensor network by genetic algorithm based deployment," in Sixth International Conference, Digital Information and Communication Technology and its Applications (DICTAP), Konya, Turkey, July 2016.

[3] S. S. Kashi, "Area coverage of heterogeneous wireless sensor networks in support of Internet of Things demands," Computing, vol. 101, no. 1-23, pp. 363-385, 2019.

[4] J. He, Z. Xing, R. Hu et al., "Directional antenna intelligent coverage method based on traversal optimization algorithm," Computers, Materials \& Continua, vol. 60, no. 2, pp. 527544, 2019.

[5] A. Ndam Njoya, W. Abdou, A. Dipanda, and E. Tonye, "Optimization of sensor deployment using multi-objective evolutionary algorithms," Journal of Reliable Intelligent Environments, vol. 2, no. 4, pp. 209-220, 2016.

[6] S. Kaur and R. Uppal, "Dynamic deployment of homogeneous sensor nodes using genetic algorithm with maximum coverage," in 2015 2nd International Conference on Computing for Sustainable Global Development (INDIACom), New Delhi, India, March 2015.

[7] A. Sangwan and R. P. Singh, "Survey on coverage problems in wireless sensor networks," Wireless Personal Communications, vol. 80, no. 4, pp. 1475-1500, 2015.

[8] M. Rout and R. Roy, "Optimal wireless sensor network information coverage using particle swarm optimisation method," International Journal of Electronics Letters, vol. 5, no. 4, pp. 491-499, 2016.

[9] C.-W. Tsai, P.-W. Tsai, J.-S. Pan, and H.-C. Chao, "Metaheuristics for the deployment problem of WSN: a review," Microprocessors and Microsystems, vol. 39, no. 8, pp. 1305-1317, 2015.

[10] T. S. Panag and J. Dhillon, "Maximal coverage hybrid search algorithm for deployment in wireless sensor networks," Wireless Networks, vol. 25, no. 2, pp. 637-652, 2019.

[11] O. Moh'd Alia and A. Al-Ajouri, "Maximizing wireless sensor network coverage with minimum cost using harmony search algorithm," IEEE Sensors Journal, vol. 17, no. 3, pp. 882-896, 2017.

[12] D. S. Deif and Y. Gadallah, "Classification of wireless sensor networks deployment techniques," IEEE Communications Surveys \& Tutorials, vol. 16, no. 2, pp. 834-855, 2014.

[13] S. M. Mohamed, H. S. Hamza, and I. A. Saroit, "Improving coverage and connectivity in mobile sensor networks using harmony search," in 2014 12th International Symposium on Modeling and Optimization in Mobile, Ad Hoc, and Wireless Networks (WiOpt), Hammamet, Tunisia, May 2014.

[14] D. Manjarres, J. Del Ser, S. Gil-Lopez, M. Vecchio, I. LandaTorres, and R. Lopez-Valcarce, "A novel heuristic approach for distance- and connectivity-based multihop node localization in wireless sensor networks," Soft Computing, vol. 17, no. 1, pp. 17-28, 2013.

[15] H. Zhang and C. Liu, "A review on node deployment of wireless sensor network," International Journal of Computer Science Issues(IJCSI), vol. 9, no. 6, p. 378, 2012. 
[16] C. Duan, J. Feng, H. Chang, J. Pan, and L. Duan, "Research on sensor network coverage enhancement based on noncooperative games," Computers, Materials \& Continua, vol. 60, no. 3, pp. 989-1002, 2019.

[17] R. Mulligan and H. M. Ammari, "Coverage in wireless sensor networks: a survey," Network protocols and algorithms, vol. 2, no. 2, pp. 27-53, 2010.

[18] Y. Miao, Y. Wang, and W. J. Xuan, "Hybrid particle swarm algorithm for minimum exposure path problem in heterogeneous wireless sensor network," International Journal of Wireless and Mobile Computing, vol. 8, no. 1, pp. 74-81, 2015.

[19] C.-H. Wu and Y.-C. Chung, "Heterogeneous wireless sensor network deployment and topology control based on irregular sensor model," in International Conference on Grid and Pervasive Computing, Lecture Notes in Computer Science, Lecture Notes in Computer Science, 2007.

[20] J. Wang, J. Chunwei, G. Yu, A. K. Sangaiah, and G.-j. Kim, “A PSO based energy efficient coverage control algorithm for wireless sensor networks," Computers, Materials \& Continua, vol. 56, no. 3, pp. 433-446, 2018.

[21] Z. W. Geem, J. H. Kim, and G. V. Loganathan, "A new heuristic optioptimizationorithm: harmony search," Simulation, vol. 76, no. 2, pp. 60-68, 2001.

[22] D. Manjarres, I. Landa-Torres, S. Gil-Lopez et al., "A survey on applications of the harmony search algorithm," Engineering Applications of Artificial Intelligence, vol. 26, no. 8, pp. 18181831, 2013.

[23] B. Alatas, "Chaotic harmony search algorithms," Applied Mathematics and Computation, vol. 216, no. 9, pp. 26872699, 2010.

[24] H. P. Gupta, Stochastic Coverage and Connectivity in Heterogeneous Wireless Sensor Networks, 2014.

[25] M. Karatas, "Optimal deployment of heterogeneous sensor networks for a hybrid point and barrier coverage application," Computer Networks, vol. 132, pp. 129-144, 2018.

[26] J.-J. Lee, B. Krishnamachari, and C.-C. Kuo, "Impact of heterogeneous deployment on lifetime sensing coverage in sensor networks," in 2004 First Annual IEEE Communications Society Conference on Sensor and Ad Hoc Communications and Networks, 2004. IEEE SECON 2004, Santa Clara, CA, USA, USA, October 2004.

[27] J.-W. Lee and W. Kim, "Design of randomly deployed heterogeneous wireless sensor networks by algorithms based on swarm intelligence," International Journal of Distributed Sensor Networks, vol. 11, no. 8, 2015.

[28] W. Abbas and X. Koutsoukos, "Efficient complete coverage through heterogeneous sensing nodes," IEEE Wireless Communications Letters, vol. 4, no. 1, pp. 14-17, 2015.

[29] S. Hu, J. Han, X. Wei, and Z. Chen, "A multi-hop heterogeneous cluster-based optimization algorithm for wireless sensor networks," Wireless Networks, vol. 21, no. 1, pp. 57-65, 2015.

[30] A. Imani and M. Eslami, "Effect of heterogeneity on coverage, energy consumption and connectivity of wireless sensor networks," in 2017 Iranian Conference on Electrical Engineering (ICEE), Tehran, Iran, May 2017.

[31] H. Kim and J. Ben-Othman, "HeteRBar: construction of heterogeneous reinforced barrier in wireless sensor networks," IEEE Communications Letters, vol. 21, no. 8, pp. 1859-1862, 2017.

[32] S. Agrawal, L. Diwan, and M. Gupta, Coverage Assessment in Randomly Deployed Wireless Sensor Networks Via Heterogeneous Nodes, Wireless Networks journal, Springer, 2015.
[33] M.-L. Lam and Y.-H. Liu, "Heterogeneous sensor network deployment using circle packings," in Proceedings 2007 IEEE International Conference on Robotics and Automation, Roma, Italy, April 2007.

[34] G. K. Brar and A. K. Virk, "Deployment of nodes for maximum coverage in heterogeneous wireless sensor network using genetic algorithm," International Journal, vol. 2, no. 6, 2014.

[35] D. T. H. Ly, N. T. Hanh, H. T. T. Binh, and N. D. Nghia, “An improved genetic algorithm for maximaximizing a coverage in wireless sensor networks," in Proceedings of the Sixth International Symposium on Information and Communication Technology, Hue City, Vietnam, 2015.

[36] D. Saha and A. Das, "Coverage area maximization heterogeneous sensor nodes with minimum displacement in mobile networks," in IEEE International Conference on Advanced Networks and Telecommuncations Systems (ANTS), Kolkata, India, 2015.

[37] C. Song, L. Liu, G. Feng, and S. Xu, "Coverage control for heterogeneous mobile sensor networks on a circle," Automatica, vol. 63, pp. 349-358, 2016.

[38] R. V. Kulkarni and G. K. Venayagamoorthy, "Particle swarm optimization in wireless-sensor networks: a brief survey," IEEE Transactions on Systems, Man, and Cybernetics, Part C (Applications and Reviews), vol. 41, no. 2, pp. 262-267, 2011.

[39] M. Akilandeswari and U. Srikanth, "Metaheuristic approach for maximizing lifetime of heterogeneous wireless sensor networks," in International Conference Computer Communication and Informatics (ICCCI), United States, 2012.

[40] Y. Lin, J. Zhang, H. S.-H. Chung, W. H. Ip, Y. Li, and Y.-H. Shi, "An ant colony optioptimizationroach for maximaximizing lifetime of heterogeneous wireless sensor networks," IEEE Transactions on Systems, Man, and Cybernetics, Part C (Applications and Reviews), vol. 42, no. 3, pp. 408-420, 2012.

[41] N. Halim, A. Isa, A. Hamid, and I. Isa, "Effect of modified harmony search towards the area coverage in wireless sensor network (WSN)," Journal of Telecommunication, Electronic and Computer Engineering (JTEC), vol. 9, no. 2-13, pp. 51-55, 2017.

[42] S. M. Mohamed, H. S. Hamza, and I. A. Saroit, "Harmony search-based k-coverage enhancement in wireless sensor networks," International Journal of Computer, Electrical, Automation, Control and Information Engineering, vol. 9, no. 1, p. 19924, 2015.

[43] A. Mohsen, W. Aljoby, K. Alenezi, and A. Alenezi, “A robust harmony search algorithm based Markov model for node deployment in hybrid wireless sensor networks," International Journal of Geomate, vol. 11, no. 27, pp. 2747-2754, 2016.

[44] A. S. Alsaidi, T.-C. Wan, and A. Munther, "Application of harmony search optimization algorithm to improve connectivity in wireless sensor network with non-uniform density," Journal of Information Science and Engineering, vol. 31, no. 4, pp. 1475-1489, 2015.

[45] S. Ebrahimnezhad, H. J. Kamali, and M. E. Moghaddam, "Extending coverage and lifetime of k-coverage wireless sensor networks using improved harmony search," Sensors \& Transducers, vol. 130, no. 7, p. 61, 2011.

[46] A. Nikdel, M. S. Kohshoori, S. M. Jamei, R. B. Ramhormoz, and I. B. Izeh, "An intelligent and energy efficient area coverage protocol for wireless sensor networks," International Journal of Grid and Distributed Computing, vol. 4, 2011.

[47] M. Nikravan and S. M. Jameii, "Combining harmony search and learning automata for topology control in wireless sensor 
networks," International Journal of Wireless \& Mobile Networks, vol. 4, no. 6, pp. 87-98, 2012.

[48] O. Moh'd Alia, Z. Shaaban, A. Basheer, A. Al-Ajouri, and A. Alsswey, "Musicians'-inspired clustering protocol for efficient energy wireless sensor networks," in Fourth International Conference on Communications and Networking, ComNet2014, Hammamet, Tunisia, March 2014.

[49] Z. Kamaei, H. Bakhshi, and B. Masoumi, "Improved harmony search algorithm with ant colony Optimization Algorithm to increase the lifetime of wireless sensor networks," International Journal of Computer Applications, vol. 120, no. 14, pp. 6-12, 2015.

[50] S. E. Nezhad, H. J. Kamali, and M. E. Moghaddam, "Solving Kcoverage problem in wireless sensor networks using improved harmony search," in 2010 International Conference on Broadband, Wireless Computing, Communication and Applications, Fukuoka, Japan, November 2010.

[51] M. A. Z. Soltani, A. T. Haghighat, H. Rashidi, and T. G. Chegini, "A couple of algorithms for k-coverage problem in visual sensor networks," in International Conference on Communication Engineering and Networks, Hong Kong, China, 2011.

[52] I. Landa-Torres, S. Gil-Lopez, J. Del Ser, S. Salcedo-Sanz, D. Manjarres, and J. A. Portilla-Figueras, "Efficient citywide planning of open WiFi access networks using novel grouping harmony searchheuristics," Engineering Applications of Artificial Intelligence, vol. 26, no. 3, pp. 1124-1130, 2013.

[53] Z.-R. Peng, H. Yin, H.-T. Dong, H. Li, and A. Pan, "A harmony search based low-delay and low-energy wireless sensor network," International Journal of Future Generation Communication and Networking, vol. 8, no. 2, pp. 21-32, 2015.

[54] J. Wang, Y. Gao, C. Zhou, R. Simon Sherratt, and L. Wang, "Optimal coverage multi-path scheduling scheme with multiple mobile sinks for WSNs," Computers, Materials \& Continua, vol. 62, no. 2, pp. 695-711, 2020.

[55] L. Wang, L. An, H.-Q. Ni, W. Ye, P. M. Pardalos, and M.R. Fei, "Pareto-based multi-objective node placement of industrial wireless sensor networks using binary differential evolution harmony search," Advances in Manufacturing, vol. 4, no. 1, pp. 66-78, 2016.

[56] Y. Sun and S. Halgamuge, "Minimum-cost heterogeneous node placement in wireless sensor networks," IEEE Access, vol. 7, pp. 14847-14858, 2019.

[57] H. Zainol Abidin and N. M. Din, "Sensor node placement in wireless sensor network based on territorial predator scent marking algorithm," ISRN Sensor Networks, vol. 2013, 7 pages, 2013.

[58] H. Zainol Abidin, N. Din, I. Yassin, H. Omar, N. Radzi, and S. Sadon, "Sensor node placement in wireless sensor network using multi-objective territorial predator scent marking algorithm," Arabian Journal for Science and Engineering, vol. 39, no. 8, pp. 6317-6325, 2014.

[59] Y. Yoon and Y.-H. Kim, "An efficient genetic algorithm for maximum coverage deployment in wireless sensor networks," IEEE Transactions on Cybernetics, vol. 43, no. 5, pp. 14731483, 2013.

[60] B. Wang, "Coverage problems in sensor networks: a survey," ACM Computing Surveys (CSUR), vol. 43, no. 4, p. 32, 2011.

[61] S. Panov and S. Koceski, "Area coverage in wireless sensor network by using harmony search algorithm," in 2014 3rd Mediterranean Conference on Embedded Computing (MECO), Budva, Montenegro, June 2014.
[62] Y. Zou and K. Chakrabarty, "Sensor deployment and target localizationed on virtual forces," in (2003) IEEE Societies, INFOCOM 2003. Twenty-Second Annual Joint Conference of the IEEE Computer and Communications, San Francisco, CA, USA, April 2003.

[63] X. Wang and S. Wang, "Hierarchical deployment optimization for wireless sensor networks," IEEE Transactions on Mobile Computing, vol. 10, no. 7, pp. 1028-1041, 2011.

[64] X.-S. Yang, "Harmony search as a metaheuristic algorithm," in Music-Inspired Harmony Search Algorithm, pp. 1-14, Springer, 2009. 